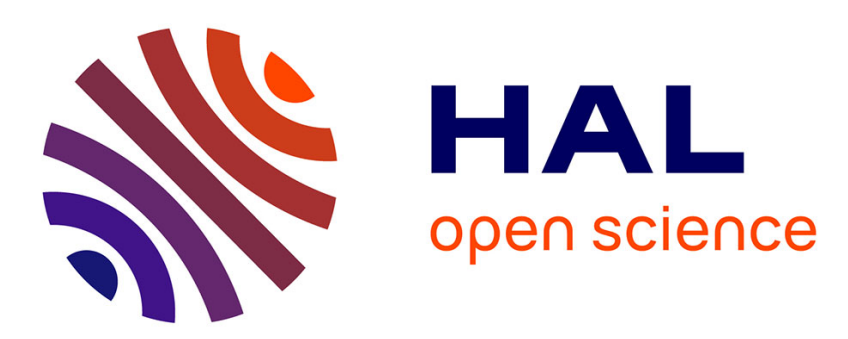

\title{
Dynamo Action in the Steeply Decaying Conductivity Region of Jupiter-Like Dynamo Models
}

\author{
Johannes Wicht, Thomas Gastine, Lucia D. V. Duarte
}

\section{To cite this version:}

Johannes Wicht, Thomas Gastine, Lucia D. V. Duarte. Dynamo Action in the Steeply Decaying Conductivity Region of Jupiter-Like Dynamo Models. Journal of Geophysical Research. Planets, 2019, 124 (3), pp.837-863. 10.1029/2018JE005759 . hal-02125041

\section{HAL Id: hal-02125041 \\ https://hal.science/hal-02125041}

Submitted on 29 Mar 2021

HAL is a multi-disciplinary open access archive for the deposit and dissemination of scientific research documents, whether they are published or not. The documents may come from teaching and research institutions in France or abroad, or from public or private research centers.
L'archive ouverte pluridisciplinaire HAL, est destinée au dépôt et à la diffusion de documents scientifiques de niveau recherche, publiés ou non, émanant des établissements d'enseignement et de recherche français ou étrangers, des laboratoires publics ou privés. 
JGR Planets

\section{RESEARCH ARTICLE \\ 10.1029/2018JE005759}

Key Points:

- Numerical simulations of Jupiter's dynamo are analyzed, concentrating on the outer steeply decaying conductivity region

- We find that the dynamo dynamics in this region is dominated by Ohmic dissipation and can thus be reasonably estimated

- The estimates can be applied to Jupiter or other planets, to reveal the possible dynamo action of zonal winds or other flow contributions

Correspondence to:

J. Wicht,

wicht@mps.mpg.de

Citation:

Wicht, J., Gastine, T., \&

Duarte, L. D. V. (2019). Dynamo

action in the steeply decaying conductivity region of Jupiter-like dynamo models. Journal of Geophysical

Research: Planets, 124, 837-863.

https://doi.org/10.1029/2018JE005759

Received 10 AUG 2018

Accepted 20 FEB 2019

Accepted article online 24 FEB 2019

Published online 28 MAR 2019

Author Contributions

Conceptualization: J. Wicht

Funding Acquisition: J. Wicht

Methodology: J. Wicht, T. Gastine, L.

D. V. Duarte

Software: J. Wicht, T. Gastine

Validation: J. Wicht, T. Gastine, L. D.

V. Duarte

Writing - Original Draft: J. Wicht

Formal Analysis: J. Wicht

Investigation: J. Wicht, T. Gastine, L. D. V. Duarte

Project Administration: J. Wicht

Resources: J. Wicht

Supervision: J. Wicht

Visualization: J. Wicht

Writing - review \& editing: J. Wicht

(C)2019. American Geophysical Union. All Rights Reserved.

\section{Dynamo Action in the Steeply Decaying Conductivity Region of Jupiter-Like Dynamo Models}

\author{
J. Wicht ${ }^{1}(\mathbb{D})$, T. Gastine ${ }^{2}$, and L. D. V. Duarte ${ }^{3}$ \\ ${ }^{1}$ Max Planck Institute for Solar System Research, Göttingen, Germany, ${ }^{2}$ IPGP, Institut de Physique du Globe de Paris, \\ Sorbonne Paris Cité, Université Paris-Diderot, Paris, France, ${ }^{3}$ College of Engineering, Mathematics and Physical \\ Sciences, University of Exeter, Exeter, UK
}

\begin{abstract}
The Juno mission is delivering spectacular data of Jupiter's magnetic field, while the gravity measurements finally allow constraining the depth of the winds observed at cloud level. However, to which degree the zonal winds contribute to the planet's dynamo action remains an open question. Here we explore numerical dynamo simulations that include a Jupiter-like electrical conductivity profile and successfully model the planet's large-scale field. We concentrate on analyzing the dynamo action in the Steeply Decaying Conductivity Region (SDCR) where the high conductivity in the metallic Hydrogen region drops to the much lower values caused by ionization effects in the very outer envelope of the planet. Our simulations show that the dynamo action in the SDCR is strongly ruled by diffusive effects and is therefore quasi-stationary. The locally induced magnetic field is dominated by the horizontal toroidal field, while the locally induced currents are dominated by the latitudinal component. The simple dynamics can be exploited to yield estimates of surprisingly high quality for both field and currents. These could potentially be exploited to predict the dynamo action of the zonal winds in Jupiter's SDCR but also in other planets.
\end{abstract}

Plain Language Summary The Juno mission is delivering spectacular data of Jupiter's magnetic field, while the gravity measurements finally allow constraining the depth of the winds observed at cloud level. However, to which degree the zonal winds contribute to the planet's dynamo action remains an open question. Here we explore numerical simulations of Jupiter's internal dynamics that successfully model the planet's large-scale field. We concentrate on analyzing the dynamo action in the Steeply Decaying Conductivity Region (SDCR) where the high conductivity in the metallic Hydrogen region drops to the much lower values in the outer envelope of the planet. Our simulations show that the dynamo action in the SDCR is strongly ruled by diffusive effects and is therefore rather simple. The simple dynamics can be exploited to yield estimates of surprisingly high quality for both the induced field and the electric currents in the SDCR. These will allow predicting the dynamo action of the zonal winds in Jupiter's SDCR but also in other planets.

\section{Introduction}

The measurements performed by the Juno mission provide very valuable constraints on the planets interior structure and dynamics. A meaningful interpretation of these data, however, requires a better understanding of the underlying physics, in particular of the dynamo process and the zonal wind dynamics. Ab initio calculations (French et al., 2012) show that the electrical conductivity first increases with depth at a superexponential rate due to hydrogen ionization. At about $0.90 r_{J}$, where $r_{J}$ is Jupiter's mean radius at the one bar level, hydrogen undergoes a transition from the molecular to a metallic state, and the conductivity increases much more smoothly with depth (see Figure 1).

It is commonly thought that Jupiter's dynamo operates in the deeper metallic region, while the fierce zonal wind system observed on the surface is limited to the molecular outer shell. However, recent numerical simulations show that the interaction between both regions yields complex and interesting dynamics. For example, the zonal winds may drive a secondary dynamo where they reach down to significant enough electrical conductivities. Possible features that could reveal such a process are banded structures and large-scale spots at low to middle latitudes (Duarte et al., 2018; Gastine, Wicht, et al., 2014), very similar to what has recently been observed by NASA's Juno mission (Connerney et al., 2018; Moore et al., 2018). 

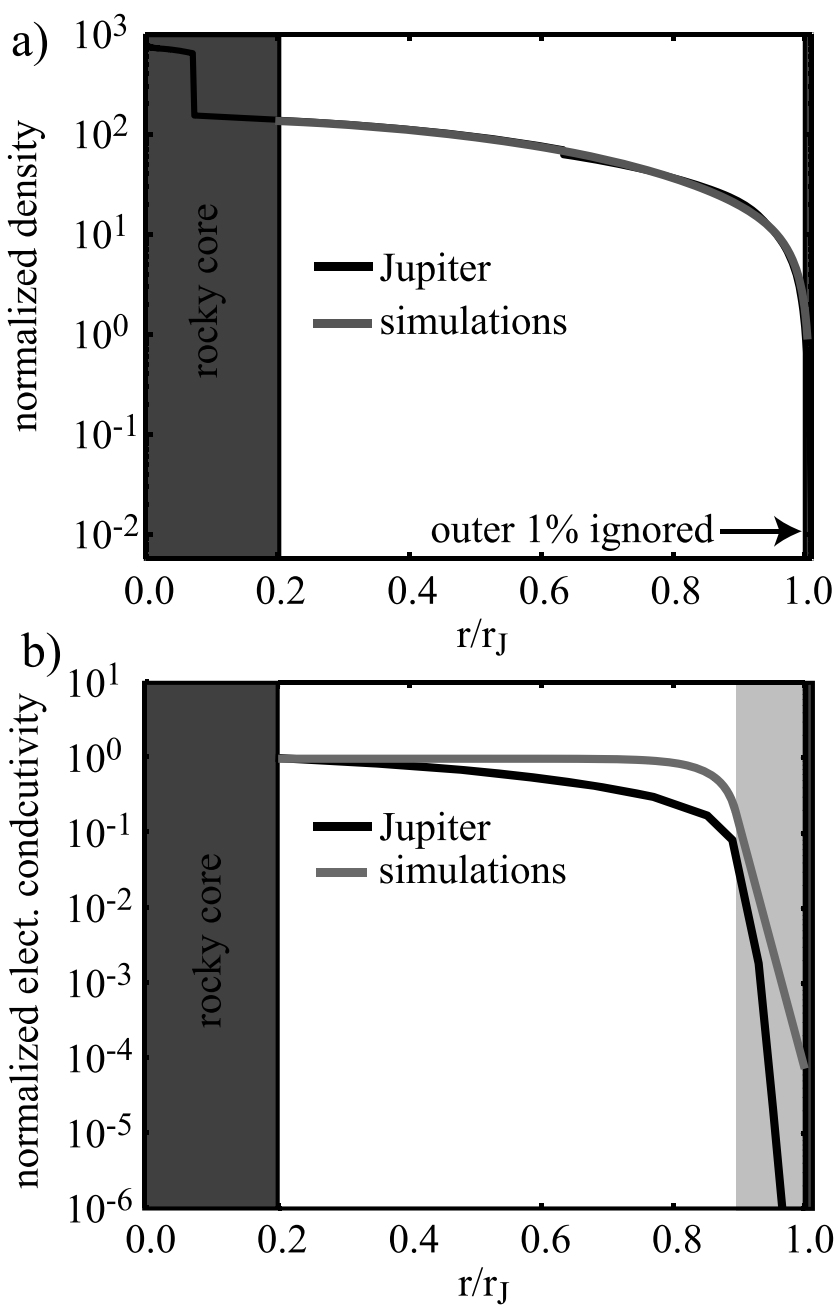

Figure 1. Comparison of the Jupiter interior model by French et al. (2012) and Nettelmann et al. (2012) (black lines) with the model used for the numerical simulations (gray). (a) and (b) show the normalized background density and electrical conductivity, respectively. Dark gray background colors mark the outer $1 \%$ that have been neglected in the simulations and the assumed solid core. Note that the core occupies $20 \%$ in radius in the numerical models but only $10 \%$ in the Jupiter model (black density profile). Medium gray highlights the Steeply Decaying Conductivity Region.

One of the main Juno objectives is to determine the depth of the fierce zonal winds observed on the planet's surface. Since the wind dynamics is tied to density variations, the gravity signal can help to constrain their depth. A recent analysis by Kaspi et al. (2018), based on the equatorially antisymmetric gravity moments $J_{3}$ to $J_{9}$ measured by the Juno mission (Iess et al., 2018), concludes that the wind speed must be significantly reduced at about 3,000 km depth, which corresponds to about $0.96 r_{J}$. This conclusion is supported by new interior models based on Juno data (Guillot et al., 2018). Kong et al. (2018), however, point out that the problem is nonunique and that the gravity signal could also be explained by deeper reaching but weaker zonal flows that may not reflect zonal jets observed at cloud level. Another hint on the depth of the zonal winds comes from the width of the prograde equatorial jet. Numerical simulations show a dependence on the depth of the spherical shell the winds live in and suggest a lower boundary at about $0.95 r_{J}$ (Gastine, Heimpel, \& Wicht, 2014).

Other estimates of the wind depth rely on magnetic effects. Liu et al. (2008) concluded that winds cannot reach deeper than $0.96 r_{J}$ with undiminished speed since the Ohmic heating produced by the zonal-wind-induced electric currents would otherwise exceed the heat emitted from the planet's interior. Ridley and Holme (2016) argue that the secular variation of the large-scale field, deduced from pre-Juno measurements, is so small that the zonal winds cannot contribute. The winds are thus unlikely to penetrate 
deeper than about $0.96 r_{J}$ where the magnetic Reynolds number exceeds unity (Cao \& Stevenson, 2017; Liu et al., 2008).

Classically, the magnetic Reynolds number attempts to quantify the ratio of magnetic induction to Ohmic dissipation in the induction equation

$$
\frac{\partial \mathbf{B}}{\partial t}=\nabla \times(\mathbf{U} \times \mathbf{B})-\nabla \times(\lambda \nabla \times \mathbf{B})
$$

where $\lambda=1 /(\mu \sigma)$ is the magnetic diffusivity with $\sigma$ the electrical conductivity and $\mu$ the magnetic permeability. The Ohmic dissipation term can be separated into two parts:

$$
\nabla \times(\lambda \nabla \times \mathbf{B})=-\lambda \nabla^{2} \mathbf{B}+\frac{\lambda}{d_{\lambda}} \hat{\mathbf{r}} \times(\nabla \times \mathbf{B}),
$$

where the second part depends on the magnetic diffusivity scale height $d_{\lambda}=\lambda /(\partial \lambda / \partial r)$. The standard magnetic Reynolds number,

$$
\mathrm{Rm}=\frac{\mathrm{U} d}{\lambda},
$$

ignores the second term. Here $d$ is a reference length scale and $U$ a typical flow velocity. Liu et al. (2008) argue that the appropriate definition for the region where the electrical conductivity decays steeply should be based on the second term in equation (2) and must therefore include the diffusivity scale height $d_{\lambda}$ :

$$
\mathrm{Rm}^{(1)}=\frac{\mathrm{U} d_{\lambda}}{\lambda}
$$

In Jupiter, $\mathrm{Rm}^{(1)}$ would characterize a Steeply Decaying Conductivity Region (SDCR) that roughly coincides with the molecular outer envelope where ionization effects determine the electrical conductivity.

For a self-consistent dynamo to operate, the overall field production has to overcome diffusion. The mean global magnetic Reynolds number for the whole dynamo region should thus exceed a critical value $\mathrm{Rm}_{c}=1$. Since the definition of Rm ignores many complexities of the dynamo process, the exact critical value is always higher and can only be determined by experiments. Christensen and Aubert (2006) explore a suite of Boussinesq dynamo simulations with rigid flow boundary conditions and constant conductivity and find self-consistent dynamo action when the Root Mean Square $(\mathrm{rms})$ magnetic Reynolds number exceeds $\mathrm{Rm}_{c} \approx$ 50.

The radius $r_{D}$ where the magnetic Reynolds number exceeds $\mathrm{Rm}_{c}$ indicates the "top of the dynamo region" that could potentially host self-consistent dynamo action. Gastine, Wicht, et al. (2014) and Duarte et al. (2018) use $\mathrm{Rm}_{c} \approx 50$ in combination with the classical $\mathrm{Rm}$ definition (3) and show that their numerical simulations produce Jupiter-like magnetic fields when $r_{D} \geq 0.9 r_{J}$. Extrapolating their findings to Jupiter conditions, using the conductivity profile suggested by French et al. (2012) and estimates for the rms nonaxisymmetric flow velocity, suggests that $r_{D} \approx 0.95 r_{J}$ (Duarte et al., 2018).

There are several reasons why these considerations are problematic. For one, self-consistent dynamo action and the related critical magnetic Reynolds number are defined in a mean sense for the whole dynamo region. The local dynamo mechanism at any given radius, however, also includes the modification of the field produced elsewhere. Another fundamental problem is that the magnetic Reynolds number actually ceases to provide a decent proxy for the ratio of induction to diffusion in the SDCR where the magnetic field approaches a diffusion-less potential field.

A more useful definition of the top of the dynamo region would be the depth where the Locally Induced Field (LIF) becomes a significant fraction of the total field. Cao and Stevenson (2017) suggest that $\mathrm{Rm}^{(1)}$ takes on a different role in the SDCR and may allow to quantify the ratio of the LIF $\hat{\mathbf{B}}$ to the background field $\tilde{\mathbf{B}}$. Using a simplified mean field dynamo model, they conclude that $\hat{\mathbf{B}}$ could reach $1 \%$ of the background field in the SDCR.

In this article we closely analyze the dynamo action in the SDCR for two different numerical dynamo simulations that both yield Jupiter-like large-scale magnetic fields. After presenting the numerical dynamo model in section 2, we analyze the simulation results in section 3. Section 4 is devoted to estimates for the locally induced electric currents and fields that allow predicting the dynamo action in the SDCR of planets. The paper closes with a discussion and conclusions in section 5 . 


\section{Numerical Dynamo Model}

\subsection{Fundamental Equations}

The numerical simulations were performed with the magneto-hydrodynamics code MagIC, which is freely available on GitHub (https://github.com/magic-sph/magic). MagIC solves for small disturbances around an adiabatic, hydrostatic background state that only depends on radius. Using the anelastic approximation for the Navier-Stokes equation allows incorporating a background density profile while filtering out fast sound waves.

The background state is based on the Jupiter model by French et al. (2012) and Nettelmann et al. (2012), who use ab initio simulations to determine the equation of states for Hydrogen and Helium and to calculate the transport properties. Figure 1 shows the normalized density profile $\tilde{\rho}(r)$ and the normalized electrical conductivity profile $\tilde{\sigma}(r)$ along with the respective profiles that have been assumed in the simulations. The transition to a metallic hydrogen state takes place at about $0.9 r_{J}$. Jupiter's SDCR thus roughly occupies the outer $10 \%$ in radius where the conductivity decays increasingly rapidly with radius, eventually reaching superexponential rates.

The numerical models disregard the outer $1 \%$ in radius where the density gradient is steepest and thus very difficult to resolve numerically. The conductivity profile used in the simulations is a combination of a polynomial branch

$$
\sigma=\sigma_{i}-\left(\sigma_{i}-\sigma_{m}\right)\left(\frac{r-r_{i}}{r_{m}-r_{i}}\right)^{a} \text { for } r<r_{m}
$$

and an exponential branch

$$
\sigma=\sigma_{m} \exp \left(a \frac{r-r_{m}}{r_{m}-r_{i}} \frac{\sigma_{m}-\sigma_{i}}{\sigma_{m}}\right) \text { for } r \geq r_{m}
$$

Here $\sigma_{i}$ is the reference conductivity at the bottom boundary $r_{i}, a$ is the exponential decay rate, and $r_{m}$ the transition radius where both branches meet with $\sigma\left(r_{m}\right)=\sigma_{m}$. The profiles in the dynamo simulations G14 and D18 use $r_{r}=r_{i}=0.2 r_{J}, a=13, r_{m}=0.9$, and $\sigma_{m}=\sigma_{r} / 5$.

While the ab initio simulations suggest that the conductivity already slowly decreases in the metallic region, the numerical simulations assume a constant value here. This keeps the magnetic Reynolds number at amplitudes that allow for dynamo action throughout this region (Duarte et al., 2018). The conductivity also decreases slower in the outer region than suggested by French et al. (2012) to ease the numerical calculations. The total conductivity contrasts are thus smaller than in the Jupiter model but still reach about four orders of magnitude. In the numerical simulations, the SDCR is roughly identical to the exponential branch.

The mathematical formulation of the problem has been extensively discussed elsewhere (Jones et al., 2011; Wicht et al., 2018). Detailed information can also be found in the online MagIC manual (http://magic-sph.github.io/). Here we only briefly introduce the essential equations.

MagIC solves the nondimensional Navier-Stokes equation

$$
\begin{aligned}
\mathrm{E} \tilde{\rho} \frac{\mathrm{d} \mathbf{U}}{\mathrm{d} t} & =-\tilde{\rho} \nabla\left(\frac{p}{\tilde{\rho}}\right)+2 \tilde{\rho} \mathbf{U} \times \hat{\mathbf{z}}-\frac{\mathrm{Ra}^{\prime} \mathrm{E}}{\operatorname{Pr} \mathrm{Di}} \tilde{\rho} \frac{\partial \tilde{T}}{\partial r} s \hat{\mathbf{r}} \\
& +\frac{1}{\mathrm{Pm}_{i}}(\nabla \times \mathbf{B}) \times \mathbf{B}+\mathrm{E} \tilde{\rho} \mathbf{F}_{v},
\end{aligned}
$$

the heat equation

$$
\tilde{\rho} \tilde{T} \frac{\mathrm{d} s}{\mathrm{~d} t}=\frac{1}{\operatorname{Pr}} \nabla \cdot(\rho \tilde{T} \nabla s)+\frac{\operatorname{Pr} \mathrm{Di}}{\mathrm{Ra}^{\prime}}\left(q_{\nu}+\frac{1}{\mathrm{Pm}_{i}^{2} \mathrm{E}} q_{J}\right)+q_{s},
$$

the induction equation

$$
\frac{\partial \mathbf{B}}{\partial t}=\nabla \times(\mathbf{U} \times \mathbf{B})-\frac{1}{\mathrm{Pm}_{i}} \nabla \times(\tilde{\lambda} \nabla \times \mathbf{B}),
$$

the continuity equation

$$
\nabla \cdot(\tilde{\rho} \mathbf{U})=0,
$$


and the solonoidal condition for the magnetic field

$$
\nabla \cdot \mathbf{B}=0 .
$$

Here $p$ is a modified pressure that includes centrifugal effects and accounts for disturbances in the gravity potential. Buoyancy variations are formulated in terms of the specific entropy $s$. Using the gradient of the normalized background temperature $\tilde{T}$ in the buoyancy term guarantees a consistent background gravity (Wicht et al., 2018). The three volumetric heating terms in equation (8) are viscous heating $q_{v}$, Joule or Ohmic heating $q_{J}$, and secular cooling $q_{s}$ (Jones, 2014; Wicht et al., 2018).

The dimensionless parameters ruling the system are the Ekman number

$$
\mathrm{E}=\frac{v}{\Omega d^{2}},
$$

the modified Rayleigh number

$$
\mathrm{Ra}^{\prime}=\frac{\alpha_{o} \tilde{g}_{o} d^{3} \tilde{T}_{o}}{\nu \kappa_{S}} \frac{s_{s}}{c_{p}}=\operatorname{Ra} \frac{s_{S}}{c_{p}},
$$

the Prandtl number

$$
\operatorname{Pr}=\frac{v}{\kappa_{S}},
$$

and the inner-boundary magnetic Prandtl number

$$
\operatorname{Pm}_{i}=\frac{v}{\lambda_{i}}
$$

Here $v$ is the (homogeneous) kinematic viscosity, $\Omega$ the rotation rate, $\alpha_{o}$ the outer-boundary thermal expansivity, $\tilde{g}_{o}$ the outer-boundary gravity, $\tilde{T}_{o}$ the outer boundary background temperature, $d=r_{o}-r_{i}$ the shell thickness, $\kappa_{S}$ the entropy diffusivity, $s_{s}$ the entropy scale, $c_{p}$ the heat capacity, $\lambda_{i}=1 /\left(\mu_{0} \sigma_{i}\right)$ the inner-boundary magnetic diffusivity, $\hat{\mathbf{r}}$ the radial unit vector, and $\hat{\mathbf{z}}$ the unit vector in the direction of the rotation axis. The aspect ratio, another dimensionless parameter, has been fixed to $r_{i} / r_{o}=0.2$.

The modified Rayleigh number Ra' is the product of the classical Rayleigh number Ra and the dimensionless entropy scale $s_{s} / c_{p}$. The dissipation number,

$$
\mathrm{Di}=\frac{d}{\tilde{T}_{o}}\left(\frac{\partial \tilde{T}_{o}}{\partial r}\right)=\frac{d \alpha_{o} \tilde{g}_{o}}{c_{p}},
$$

is defined by the background temperature profile $\tilde{T}$.

The equations have been nondimensionalized by using the imposed entropy difference as entropy scale $s_{s}$, $d$ as a length scale, the viscous diffusion time $d^{2} / v$ as a time scale, and $\sqrt{\Omega \mu_{0} \lambda_{i} \tilde{\rho}_{o}}$ as a magnetic scale, where $\tilde{\rho}_{o}$ is the outer-boundary reference density. We use entropy rather than temperature diffusion in the heat equation, which considerably simplifies the system (Braginsky \& Roberts, 1995; Lantz \& Fan, 1999). In the above formulation, the dimensionless profiles $\tilde{\rho}, \tilde{T}$, and $\tilde{\lambda}$ carry the information on the radial dependence of the background state.

\subsection{Poloidal/Toroidal Decomposition}

We use the common representation of the divergence free magnetic field by a poloidal and a toroidal contribution,

$$
\mathbf{B}=\mathbf{B}^{(\mathrm{P})}+\mathbf{B}^{(\mathrm{T})}=\nabla \times \nabla \times \hat{\mathbf{r}} b^{(\mathrm{P})}+\nabla \times \hat{\mathbf{r}} b^{(\mathrm{T})},
$$

where $b^{(\mathrm{P})}$ is the poloidal and $b^{(\mathrm{T})}$ the toroidal scalar potential. The respective decomposition for the electric current density $\mathbf{j}$ reads

$$
\mathbf{j}=\mathbf{j}^{(\mathrm{P})}+\mathbf{j}^{(\mathrm{T})}=\nabla \times \nabla \times \hat{\mathbf{r}} i^{(\mathrm{P})}+\nabla \times \hat{\mathbf{r}} i^{(\mathrm{T})},
$$


Table 1

Parameters of the Numerical Dynamo Simulations Explored Here

\begin{tabular}{lcccc}
\hline Name & $\mathrm{E}$ & $\mathrm{Ra}$ & $\mathrm{Pm}$ & $\mathrm{Pr}$ \\
\hline D18 & $10^{-4}$ & $6 \times 10^{7}$ & 2 & 0.1 \\
G14 & $10^{-5}$ & $5 \times 10^{9}$ & 0.6 & 1 \\
\hline
\end{tabular}

Note. Both simulations use background density and temperature models very close to those suggested by Nettelmann et al. (2012) and the electrical conductivity profile illustrated in Figure 1.

where $i^{(\mathrm{P})}$ denotes the poloidal and $i^{(\mathrm{T})}$ the toroidal potential.

Simple vector calculus yields

$$
\mathbf{j}=-\nabla_{H}^{2} i^{(\mathrm{P})} \hat{\mathbf{r}}+\nabla_{H} \frac{\partial}{\partial r} i^{(\mathrm{P})}+\nabla \times \hat{\mathbf{r}} i^{(\mathrm{T})}
$$

where $\nabla_{H}$ is the horizontal component of the nabla operator. The toroidal contribution obviously has no radial component. Radial derivatives appear only in the horizontal poloidal current density, which therefore dominates in the SDCR, as we will show below.

Ampere's law, $\nabla \times \mathbf{B}=\mu \mathbf{j}$, connects the poloidal (toroidal) magnetic field to the toroidal (poloidal) current density. Its radial component establishes a connection between toroidal current density and toroidal field potentials:

$$
\mu j_{r}=-\mu \nabla_{H}^{2} i^{(\mathrm{P})}=\hat{\mathbf{r}} \cdot(\nabla \times \mathbf{B})=-\nabla_{H}^{2} b^{(\mathrm{T})} .
$$

The toroidal field at a given radius is thus determined by the radial currents flowing through the respective radial shell.

The radial component of the curl of Ampere's law provides a relation between the toroidal current and poloidal magnetic field potentials:

$$
\begin{gathered}
\mu \hat{\mathbf{r}} \cdot(\nabla \times \mathbf{j})=-\mu \nabla_{H}^{2} i^{(\mathrm{T})}=\hat{\mathbf{r}} \cdot(\nabla \times \nabla \times \mathbf{B}) \\
=-\left(\frac{1}{r^{2}} \frac{\partial}{\partial r} r^{2} \frac{\partial}{\partial r}+\frac{2}{r^{2}}-\nabla_{H}^{2}\right) \mathrm{B}_{r} \\
=\nabla_{H}^{2}\left[\left(\frac{\partial}{\partial r}\right)^{2}+\nabla_{H}^{2}\right] b^{(P)} .
\end{gathered}
$$

\subsection{Selected Simulations}

We concentrate on closely analyzing two quite different dynamo simulations that both reproduce Jupiter's large-scale field (Duarte et al., 2018). Model G14 has been introduced by Gastine, Wicht, et al. (2014), while model D18 is listed as model number 20 in Duarte et al. (2018); Table 1 compares their parameters. Both dynamos share the background pressure, temperature, density, and electrical conductivity models (see Figure 1 for the latter two), use stress-free outer but rigid inner-boundary conditions, employ constant entropy boundary conditions, and are driven by heat coming in through the lower boundary. Model D18 uses a larger Ekman and larger magnetic Prandtl number. The Prandtl number is one in G14 but only 0.1 in D18, which yields a smaller-scale field in the latter simulation (Duarte et al., 2018).

Figure 2 compares the radial surface fields for the two snapshots which we will continue to analyze throughout the paper with the recent Jupiter field model JRM09 by Connerney et al. (2018), demonstrating that the numerical simulations at least capture Jupiter's large-scale field. A closer comparison with the older field model VIP4 (Connerney et al., 1998) can be found in Gastine, Wicht, et al. (2014) and Duarte et al. (2018). Figure 3 illustrates the flow structure for both dynamos. They share the fact that the zonal flows (left panels) show a pronounced equatorial jet but not the multitude middle to high latitude jets observed in Jupiter's or Saturn's cloud structure. These jets seem incompatible with dipole-dominated magnetic fields in the numer- 


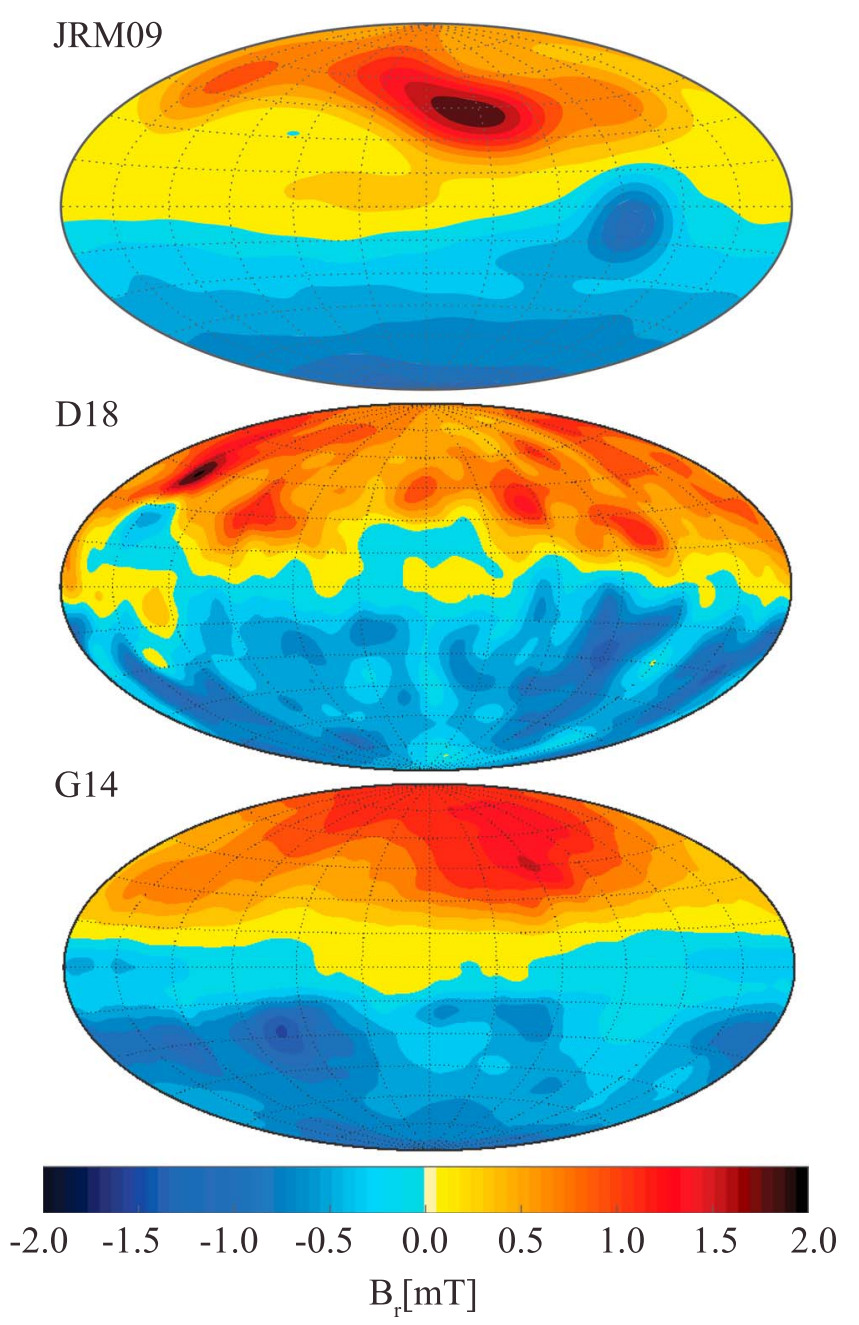

Figure 2. Comparison of the radial field in the recent Jupiter field model JRM09 (Connerney et al., 2018), plotted on a spherical surface with radius $r_{J}$, with the radial surface fields in the two snapshots of the dynamo D18 and G14 closely explored here. G14 has been rescaled to $m T$ with the power-based scaling (Duarte et al., 2018; Gastine, Wicht, et al., 2014). Because this would yield a too strong field for D18, we have scaled the field strength of the snapshot to JRM09 values for illustration purposes. Hammer projection shown.

ical simulations (Duarte et al., 2013; Gastine et al., 2012). The rms nonaxisymmetric flow amplitude (right panels in Figure 3) increases with radius because of the decreasing density (Gastine \& Wicht, 2012).

\subsection{Magnetic Reynolds Numbers}

As already discussed in section 1, the strong decrease in the electrical conductivity in the SDCR requires a modification of the classical magnetic Reynolds number. In addition to profiles following the classical definition,

$$
\mathrm{Rm}=\frac{\langle\mathbf{U}\rangle d}{\lambda}
$$

we will use

$$
\mathrm{Rm}^{(1)}=\frac{\langle\mathbf{U}\rangle d_{\lambda}}{\lambda},
$$

and

$$
\mathrm{Rm}^{(2)}=\frac{\langle\mathbf{U}\rangle d_{\lambda}^{2}}{\lambda d},
$$



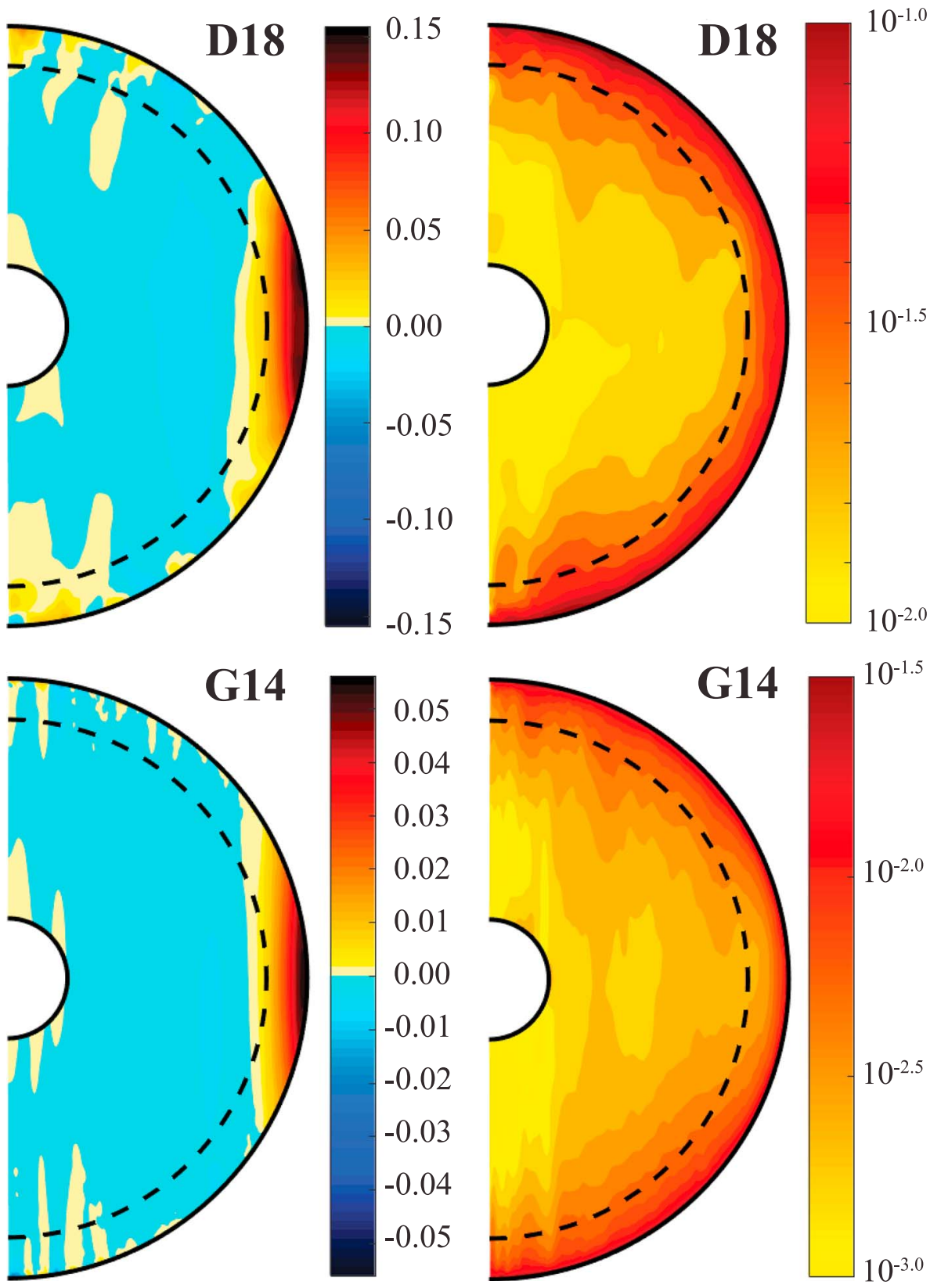

Figure 3. Zonal flows (left panels) and rms nonaxisymmetric flows (right panels) for dynamo D18 and dynamo G14 snapshots. Rossby number scales, Ro $=\mathrm{U} /(d \Omega)$, have been used here.

where $d_{\lambda}=\lambda /(\partial \lambda / \partial r)$ is the magnetic diffusivity scale height. Angular brackets generally denote spherical rms values,

$$
\langle f(r)\rangle=\left(\frac{1}{4 \pi} \int_{-1}^{1} \mathrm{~d} x \int_{0}^{2 \pi} \mathrm{d} \phi f^{2}\right)^{1 / 2},
$$

with $\phi$ being longitude, $\theta$ colatitude, and $x=\cos (\theta)$. $\langle\mathrm{U}\rangle$ thus indicates the rms velocity at a given radius.

Figure 4 compares the different magnetic Reynolds number profiles for the D18 and G14 snapshots, separating zonal flow and nonaxisymmetric flow contributions. Axisymmetric latitudinal and radial flows are typically much weaker and have thus been neglected here. While zonal and nonaxisymmetric flows have comparable amplitudes in the SDCR of dynamo D18, the zonal flows are somewhat more pronounced in model G14 because of the smaller Ekman number. In the exponential branch of the conductivity profiles, the 

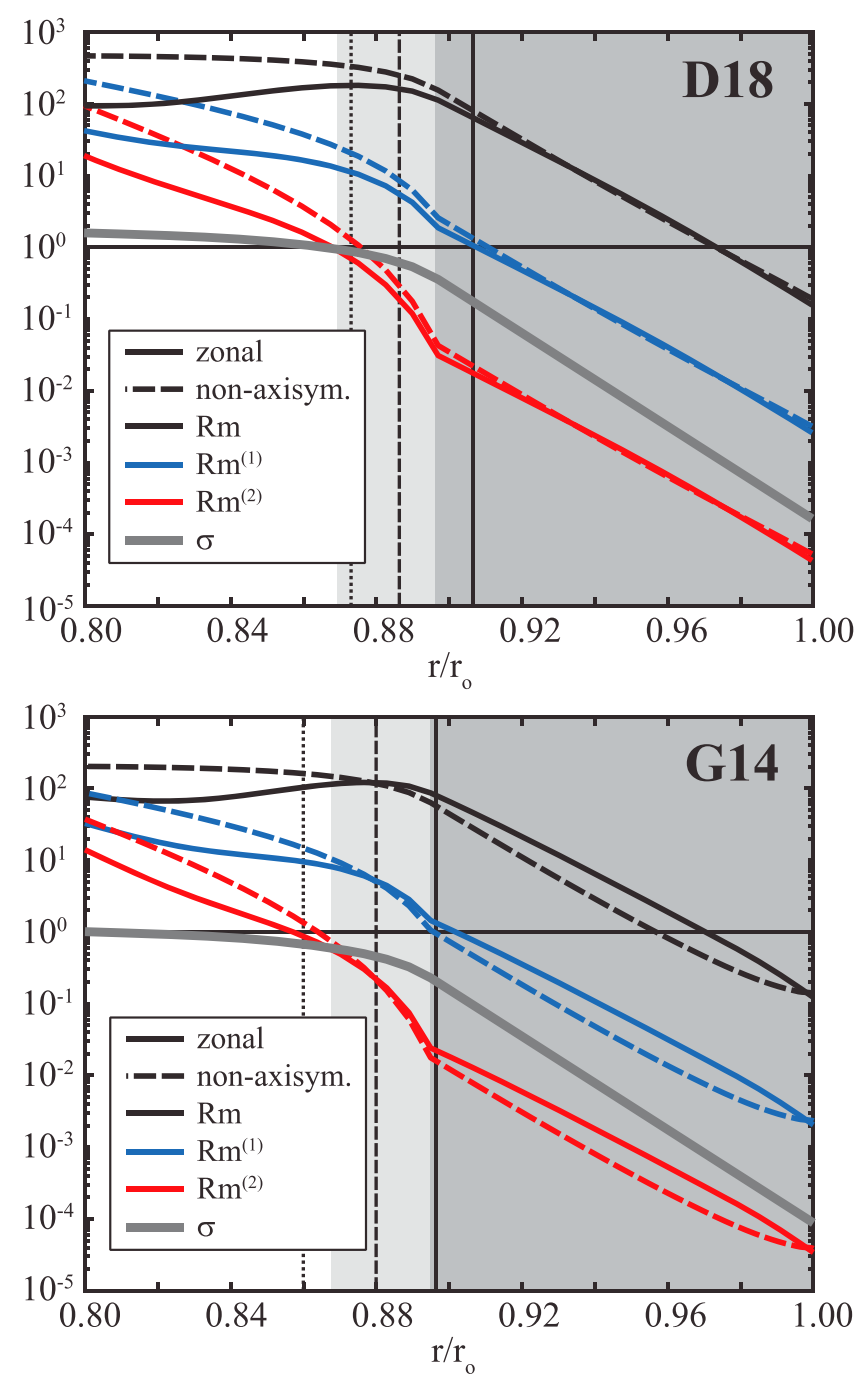

Figure 4. Radial profiles of the three different magnetic Reynolds numbers for the selected snapshots of dynamo models D18 and G14. Vertical lines mark the radii where $\mathrm{Rm}^{(1)}=1$ (solid), $\mathrm{Rm}^{(1)}=5$ (dashed), and $\mathrm{Rm}^{(2)}=1$ (dotted) for the total rms flow. A rescaled electrical conductivity profile $\sigma(r)$ (thick gray) has been include in order to demonstrates that it dominates the radial gradients in the Steeply Decaying Conductivity Region (medium gray background).

diffusivity scale height assumes a constant value of $d_{\lambda}=0.168$, which explains why the different Rm profiles are parallel in this region (see Figure 4).

\section{Dynamo Action in the SDCR}

Ohm's law,

$$
\mathbf{j}=\sigma(\mathbf{U} \times \mathbf{B}+\mathbf{E})
$$

states that the electric currents are driven by induction or by an electric field E. Since both effects scale with $\sigma$, the current density $\mathbf{j}$ has to decay in the SDCR. Figure 5 illustrates the radial decay of the different current density components for the selected snapshots in dynamos D18 (top panel) and G14 (bottom panel). The poloidal current (solid lines), more specifically its latitudinal component (solid blue), dominates in the SDCR. The rms poloidal current is at least two times larger than the toroidal current in model D18 and about five times larger than its toroidal counterpart in model G14. The likely reason for the larger difference is the stronger zonal flow in the latter simulation. Radial currents (solid black) are effectively blocked by the conductivity gradient and are thus generally small. 

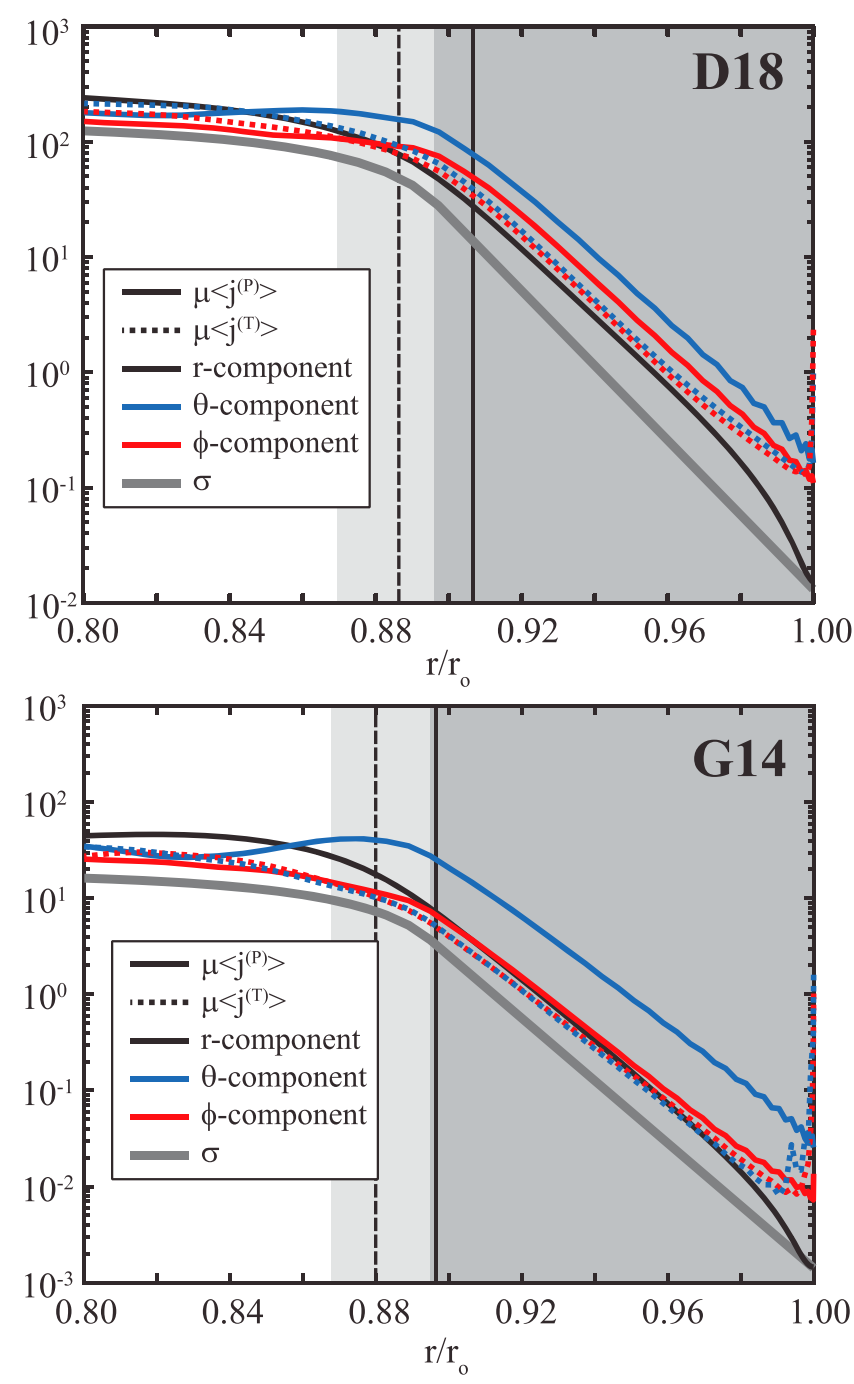

Figure 5. Radial profiles of different rms current contributions. Light gray background colors indicate where the conductivity is smaller than $50 \%$ of its reference values at $r_{i}$; medium gray highlights the Steeply Decaying Conductivity Region where the conductivity decays exponentially. Top and bottom panels show the selected snapshots for dynamos D18 and G14, respectively. Vertical lines mark the radii where $\mathrm{Rm}^{(1)}=1$ (solid) and $\mathrm{Rm}^{(1)}=5$ (dashed). A rescaled electrical conductivity profile $\sigma(r)$ has been included to demonstrate that it determines the radial gradient. Dimensionless quantities shown.

As expected, the decrease of the rms current density in the SDCR is mostly dictated by the electrical conductivity profile (thick gray line in Figure 5) with local induction effects providing some moderation. Below the SDCR, the current increases much more smoothly with depth and never exceeds twice the value reached at the bottom of the SDCR. The transition to more classical dynamo action seems to kick in somewhere between $\mathrm{Rm}^{(1)}=1$ (solid vertical line in Figure 5) and $\mathrm{Rm}^{(1)}=5$ (dashed vertical line).

In the simulations we assume that the region $r>r_{o}$ is electrically insulating. The current-free conditions, gradually approached with radius in the SDCR, are therefore abruptly enforced at $r_{0}$. Figure 5 illustrates that this leads to a thin boundary layer where the simple dependence on $\sigma$ breaks down. Since the currents are rather small in this very outer region, it also becomes increasingly difficult to calculate reliable values based on the single precision snapshots stored during the simulations.

Of particular interest is the fraction of the total field produced by the currents flowing in the SDCR. The toroidal LIF is directly given by the local poloidal currents (see equation (20)). The local poloidal field, however, is in principle produced by all the electric currents in the system. To quantify the LIF, we analyze the field $\hat{\mathbf{B}}(r)$ that is induced by the currents flowing in the region above $r$. Since the currents and thus $\hat{\mathbf{B}}(r)$ 

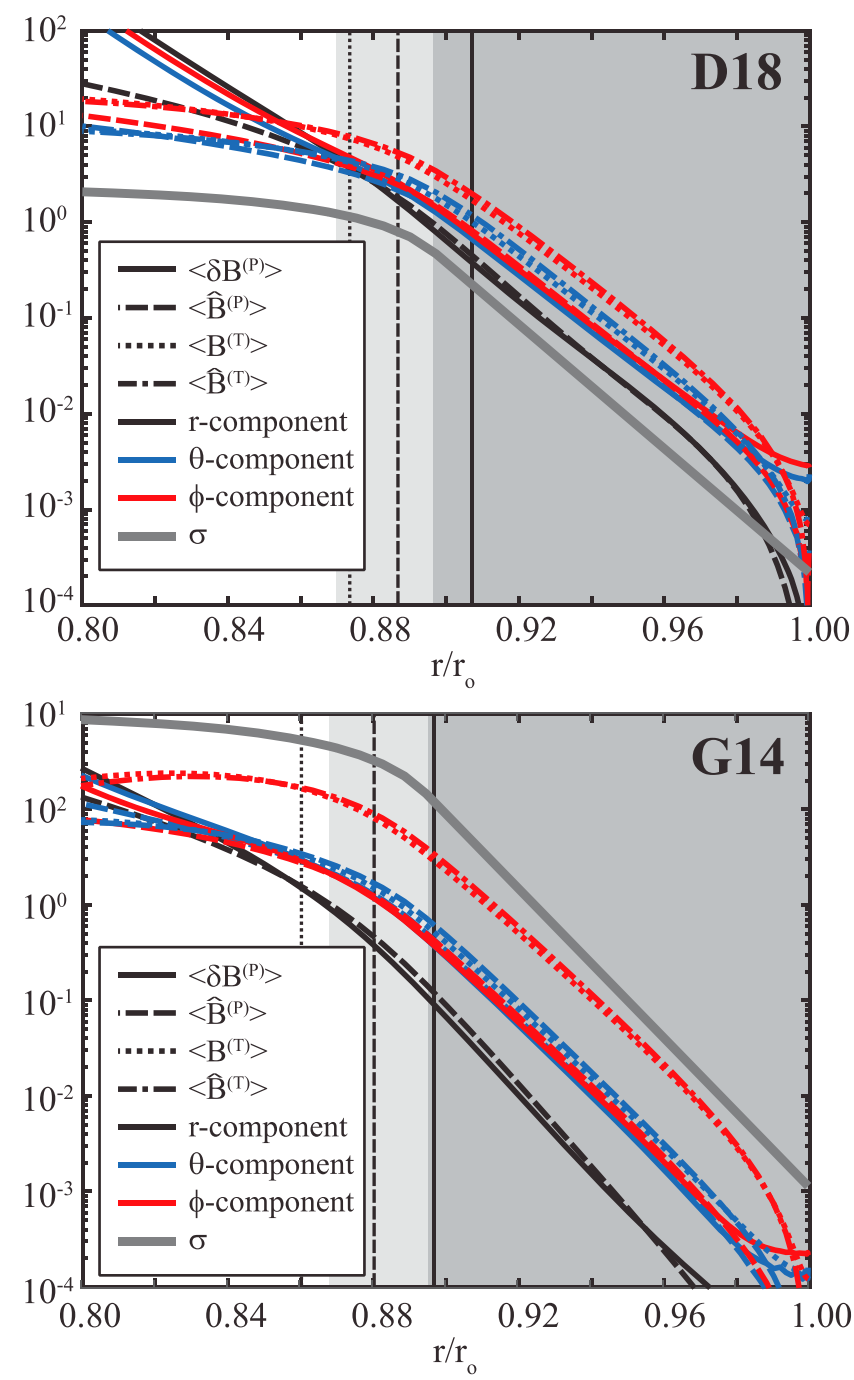

Figure 6. Comparison of the rms values from approximations (30) and (31) of the Locally Induced Field with $\delta \mathbf{B}$ and the true toroidal field. Vertical lines mark the radii where $\mathrm{Rm}^{(1)}=1$ (solid), $\mathrm{Rm}^{(1)}=5$ (dashed), and $\mathrm{Rm}^{(2)}=1$ (dotted). A rescaled electrical conductivity profile $\sigma(r)$ has been included to demonstrate that it determines the radial gradient.

steeply decrease with radius, Ampere's law simplifies to

$$
\frac{\partial \hat{\mathbf{B}}_{H}}{\partial r} \approx-\mu \hat{\mathbf{r}} \times \mathbf{j}_{H},
$$

where the index $H$ refers to the horizontal components. Integration in radius yields

$$
\hat{\mathbf{B}}_{H}(r) \approx \mu \hat{\mathbf{r}} \times \int_{r}^{r_{o}} \mathrm{~d} r^{\prime} \mathbf{j}_{H}\left(r^{\prime}\right) .
$$

Similarly, an integration of equation (21) provides an approximation for the radial LIF:

$$
\hat{B}_{r}(r) \approx-\mu \int_{r}^{r_{o}} \mathrm{~d} r^{\prime} \int_{r^{\prime}}^{r_{o}} \mathrm{~d} r^{\prime \prime} \hat{\mathbf{r}} \cdot\left[\nabla^{\prime \prime} \times \mathbf{j}^{(T)}\left(r^{\prime}\right)\right] .
$$

We have assumed here that $\hat{\mathbf{B}}$ and its radial derivative vanish at the outer boundary.

As long as the local currents are small, the downward continuation of the surface field, according to the characteristic radial dependence of a potential field, provides a decent approximation of the total poloidal field: 

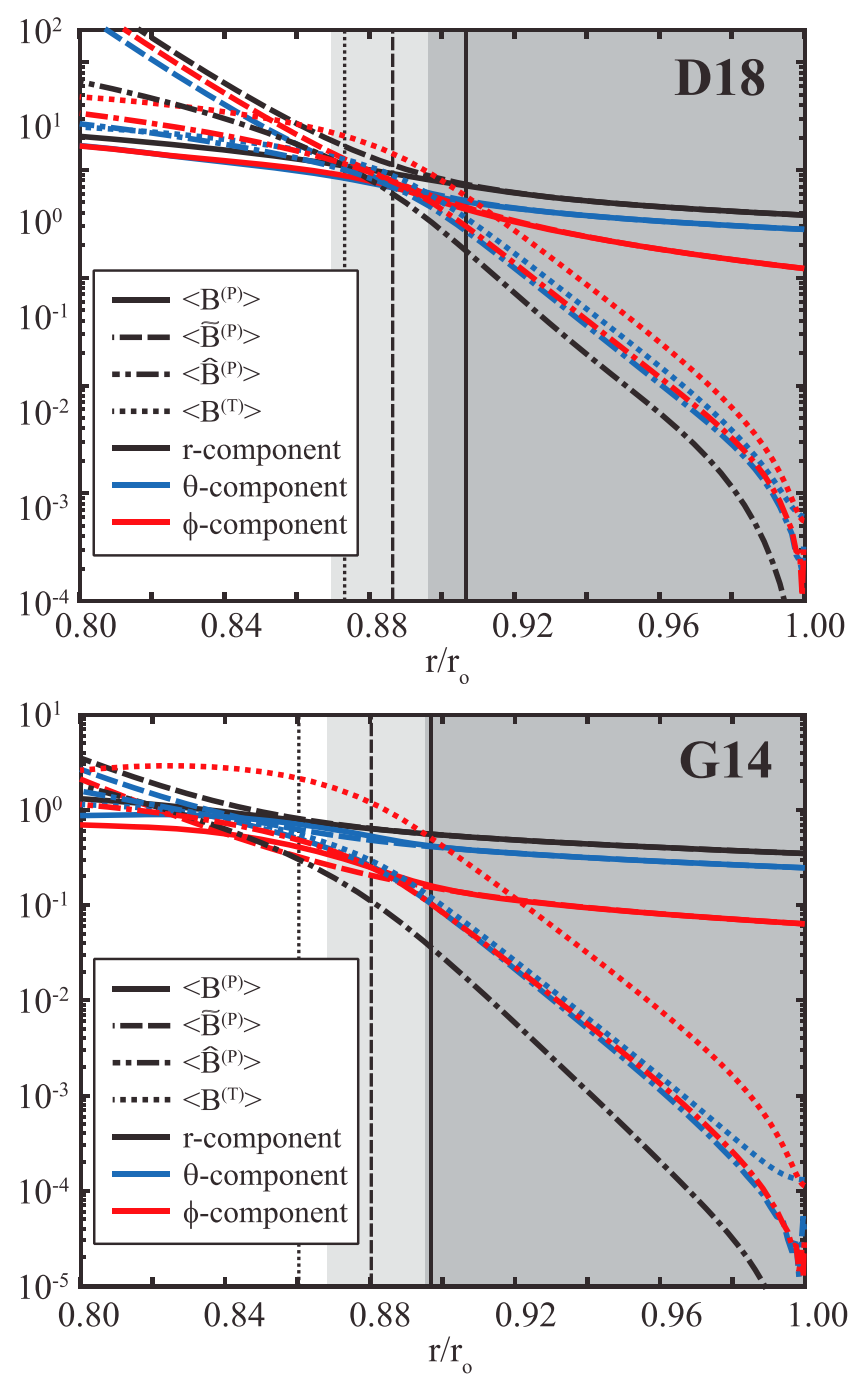

Figure 7. Rms values of different magnetic field contributions. The downward continued potential field $\tilde{\mathbf{B}}$ (dashed lines) only starts to deviate more significantly from the poloidal field $\mathbf{B}^{(P)}$ (solid lines) below the radius where $\mathrm{Rm}^{(1)}=1$ (vertical solid line). See caption of Figure 6 for more explanations.

$$
\tilde{\mathbf{B}}_{\ell}(r)=\left(\frac{r_{o}}{r}\right)^{\ell+2} \mathbf{B}_{\ell}\left(r_{o}\right) \approx \mathbf{B}^{(\mathrm{P})}(r) .
$$

We will thus use $\tilde{\mathbf{B}}(r)$ as a proxy for the background field in the SDCR dynamo process.

The difference,

$$
\delta \mathbf{B}=\mathbf{B}^{(\mathrm{P})}-\tilde{\mathbf{B}},
$$

provides a measure for the additional poloidal field produced by the local dynamo mechanism. Both $\delta \mathbf{B}$ and $\hat{\mathbf{B}}$ vanish at the outer boundary and decay radially with the electrical conductivity. Figure 6 compares rms profiles of the different $\delta \mathbf{B}$ and toroidal field contributions with those provided by approximations (30) and (31). Using the toroidal or poloidal current densities allows separating the poloidal and toroidal LIF, respectively. The close agreements confirm the high quality of the approximations. Pearson correlation coefficients are close to 1 for the toroidal horizontal components, above 0.95 for the poloidal horizontal components, and above 0.9 for the radial component in the SDCR of dynamos G14 and D18. Figure 6 demonstrates that the azimuthal toroidal field (dashed red) dominates the other LIF components, likely due to the $\Omega$ effects provided by the shearing action of the zonal winds.

Figure 7 compares the rms values of the LIF to the total poloidal field $\mathbf{B}^{(\mathrm{P})}$ and the downward continued field $\tilde{\mathbf{B}}$. The latter stops proving a reasonable approximation of the background field somewhere between the 

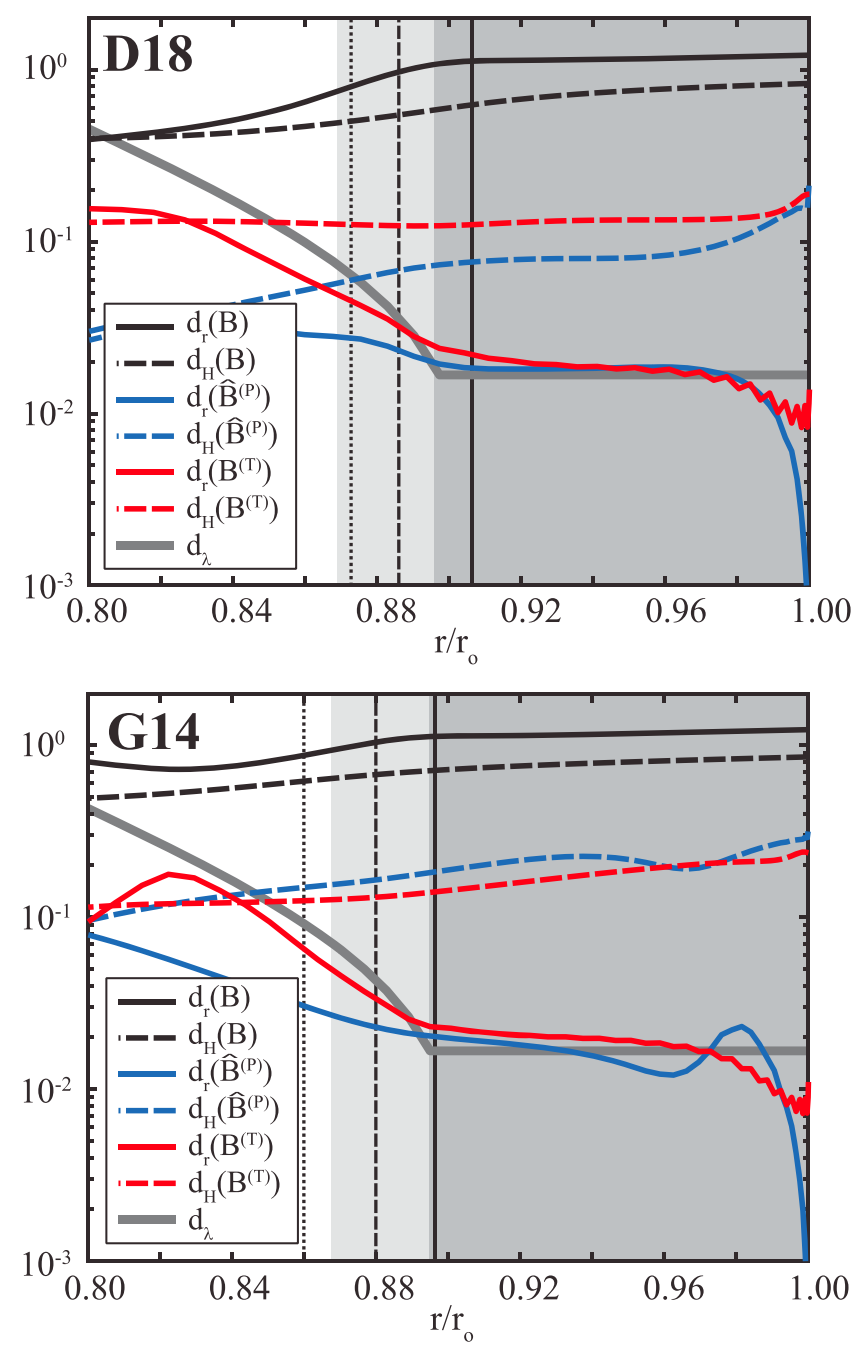

Figure 8. Radial (solid) and horizontal (dashed) rms length scales of different field contributions. See caption of Figure 6 and the main text for more explanations.

radii where $\mathrm{Rm}^{(1)}=1$ (solid vertical line) and $\mathrm{Rm}^{(1)}=5$ (dashed vertical line). The azimuthal component of the toroidal field reaches the respective background field component where $\mathrm{Rm}^{(1)} \approx 1$. We come back to discussing this issue in section 4.

Radial and horizontal magnetic field length scales, based on the respective derivatives of the field potentials, are illustrated in Figure 8. For example, the radial scales of the poloidal field are estimated via

$$
d_{r}\left(B^{(p)}\right)=\frac{\left\langle b^{(\mathrm{P})}\right\rangle}{\left\langle\partial b^{(\mathrm{P})} / \partial r\right\rangle} .
$$

Horizontal scales have been calculated using the square root of $\left\langle\nabla_{H}^{2} b\right\rangle$. Between $r=0.90 r_{o}$ and $r=0.97 r_{o}$, the radial scale of $\hat{\mathbf{B}}$ and of the toroidal field is indeed very similar to the diffusive scale height $d_{\lambda}$, which confirms that the conductivity profile determines the radial gradient of the LIF. The horizontal length scales of both LIF contributions are significantly smaller than the respective scales of the total field (or the potential field) but still an order of magnitude larger than the radial scales.

The separate evolution equations for the toroidal and poloidal field potentials solved by MagIC are the radial component of the induction equation (Christensen \& Wicht, 2007),

$$
\nabla_{H}^{2} \dot{b}^{(\mathrm{P})}=-\hat{\mathbf{r}} \cdot[\nabla \times(\mathbf{U} \times \mathbf{B})]+\lambda \nabla_{H}^{2}\left[\left(\frac{\partial}{\partial r}\right)^{2}+\nabla_{H}^{2}\right] b^{(\mathrm{P})},
$$



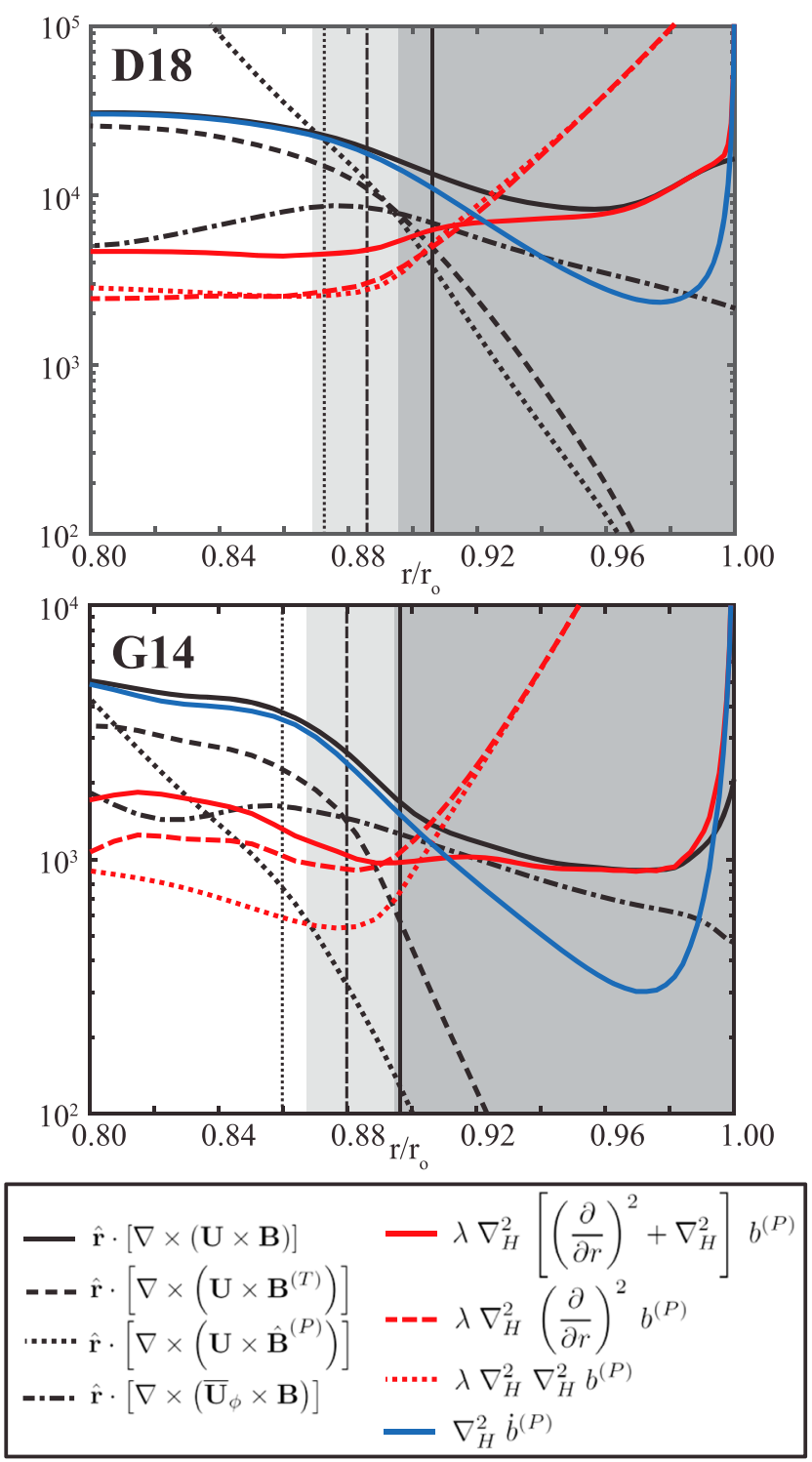

Figure 9. Horizontally averaged rms values of the different contributions to the poloidal induction equation for the snapshots in dynamos D18 (top) and G14 (bottom). See caption of Figure 6 for more explanations.

and the radial component of its curl,

$$
\nabla_{H}^{2} \dot{b}^{(\mathrm{T})}=-\hat{\mathbf{r}} \cdot[\nabla \times \nabla \times(\mathbf{U} \times \mathbf{B})]+\lambda \nabla_{H}^{2}\left[\left(\frac{\partial}{\partial r}\right)^{2}+\nabla_{H}^{2}+\frac{1}{d_{\lambda}} \frac{\partial}{\partial r}\right] b^{(\mathrm{T})} .
$$

Only the toroidal field equation directly includes the magnetic diffusivity scale height.

Figure 9 shows radial profiles of the rms values of the different contributions in the poloidal evolution equation for the D18 and G14 snapshots. Very close to the outer boundary beyond $r \approx 0.98 r_{o}$, the use of single precision snapshot data limits the quality of the second radial derivative required for the diffusive contributions. The balance should thus not be interpreted in this region.

We have separated the diffusive term into two contributions involving radial (red dashed line) and only horizontal (red dotted) derivatives. Both increase with $\lambda$ toward the outer boundary but also progressively cancel each other since the field approaches a potential field. What remains is the diffusive term for the LIF (red solid), which is mostly balanced by the induction term (black solid). Consequently, magnetic field variations (blue) become comparatively small in the highly diffusive SDCR, and the dynamics becomes quasi-stationary. 

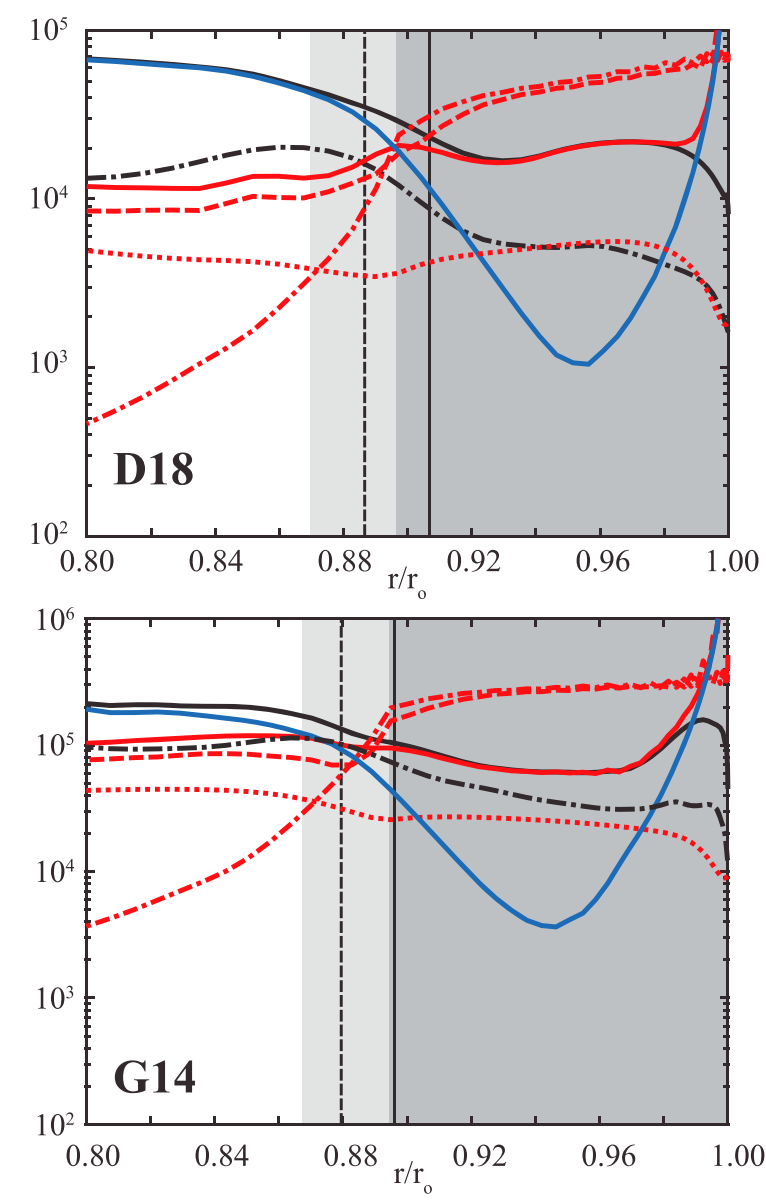

$$
\begin{array}{ll}
-\hat{\mathbf{r}} \cdot[\nabla \times \nabla \times(\mathbf{U} \times \mathbf{B})] & --\boldsymbol{\lambda} \nabla_{H}^{2}\left(\frac{\partial}{\partial r}\right)^{2} b^{(T)} \\
\mathbf{- - \boldsymbol { \mathbf { r } }} \cdot\left[\nabla \times \nabla \times\left(\overline{\mathbf{U}}_{\phi} \times \mathbf{B}\right)\right] & \cdots \cdots \lambda \nabla_{H}^{2} \nabla_{H}^{2} b^{(T)} \\
-\nabla_{H}^{2} \dot{b}^{(T)} & \cdots \cdots \nabla_{H}^{2} \frac{1}{d_{\lambda}} \frac{\partial}{\partial r} b^{(T)} \\
-\lambda \nabla_{H}^{2}\left[\left(\frac{\partial}{\partial r}\right)^{2}+\nabla_{H}^{2}+\frac{1}{d_{\lambda}} \frac{\partial}{\partial r}\right] b^{(T)}
\end{array}
$$

Figure 10. Horizontally averaged rms values of the different contributions to the toroidal induction equation for the snapshots in dynamos D18 (top) and G14 (bottom). See caption of Figure 6 for more explanations.

Figure 9 also illustrates that induction due to zonal flows (black dashed-dotted) is sizable for the poloidal field evolution. This describes pure advection of the background field $\tilde{\mathbf{B}}$. Induction due to flow acting on the toroidal field (black dashed) or the poloidal LIF (black dotted), on the other hand, remain minor contributions in the SDCR.

For the toroidal field evolution, depicted in Figure 10, time variations are even less important in the SDCR than for the poloidal counterpart. The two dominant diffusive terms that involve radial derivatives (red dashed and dash-dotted lines) cancel to a high degree. This is simply a consequence of the fact that the toroidal field decreases with $\sigma$ so that

$$
\frac{\partial}{\partial r} b^{(\mathrm{T})} \approx-\frac{b^{(\mathrm{T})}}{d_{\lambda}}
$$

Zonal flows clearly dominate the toroidal field induction, in particular for dynamo G14.

Vertical lines in Figures 9 and 10 mark where the magnetic Reynolds number $\mathrm{Rm}^{(1)}$ exceeds one (solid) and five (dashed). The assumption of quasi-stationary dynamics seems to be limited to the region $\mathrm{Rm}^{(1)} \leq 1$. 
As we will discuss below, $\mathrm{Rm}^{(2)}$ roughly quantifies the ratio of radial field induction to diffusion in the SDCR. Where $\mathrm{Rm}^{(2)}=1$, indicated by the dotted vertical line in Figure 9, we would thus expect comparable rms values of diffusion (red line) and induction (black line). This is not the case, however, since the respective radius already lies below the SDCR.

\section{Estimating the Dynamo Action}

The analysis of dynamo simulations has shown that the dynamo action in the SDCR is dominated by diffusive effects and thus obeys a quasi-stationary dynamics. In this section, we exploit this simplicity and derive expressions that could be used to estimate the dynamo action in the SDCR of planets.

\subsection{Estimating the Electric Currents}

The decent balance between diffusion and induction in the dynamo equation suggests

$$
\nabla \times \frac{\mathbf{j}}{\sigma} \approx \nabla \times(\mathbf{U} \times \tilde{\mathbf{B}}) .
$$

When using the fact that the horizontal current components dominate in the SDCR, this leads to the radial integral estimate $\mathscr{J}_{H}$ used by Liu et al. (2008):

$$
\mathbf{j}_{H} \approx \mathscr{J}_{H}(r)=\frac{\sigma}{r}\left[\frac{r^{\prime}}{\sigma} \mathbf{j}_{H}\right]_{r_{o}}+\frac{\sigma}{r} \hat{\mathbf{r}} \times \int_{r}^{r_{o}} \mathrm{~d} r^{\prime} r^{\prime}[\nabla \times(\mathbf{U} \times \tilde{\mathbf{B}})]_{H} .
$$

The square brackets with index $r_{o}$ indicate a value taken at the outer boundary. We will generally assume that the current vanishes at $r_{0}$.

A simpler estimate can be based on Ohm's law. Since the dynamics in the SDCR is to a good degree quasi-stationary, the electric field contributions due to magnetic field variations, expressed by the Maxwell-Faraday law $\partial \mathbf{B} / \partial t=-\nabla \times \mathbf{E}$, are small. Ohm's law thus reduces to

$$
\mathbf{j}=\sigma(\mathbf{U} \times \mathbf{B}-\nabla V),
$$

where $V$ is the electric potential.

Taking the radial component of the curl of Ohm's laws shows that the electric potential does not contribute to the toroidal current density:

$$
\nabla_{H}^{2} i^{(\mathrm{T})} \approx-\sigma \hat{\mathbf{r}} \cdot \nabla \times(\mathbf{U} \times \tilde{\mathbf{B}})
$$

Solving this differential equation in spectral space yields

$$
i_{\ell}^{(\mathrm{T})}=\sigma \frac{r^{2}}{\ell(\ell+1)} \hat{\mathbf{r}} \cdot[\nabla \times(\mathbf{U} \times \tilde{\mathbf{B}})]_{\ell},
$$

where the subindex $\ell$ denotes contributions of spherical harmonic order $\ell$. We will formally use the notation

$$
i^{(\mathrm{T})} \approx-\sigma \nabla_{H}^{-2} \hat{\mathbf{r}} \cdot \nabla \times(\mathbf{U} \times \tilde{\mathbf{B}}),
$$

in the following, where $\nabla_{H}^{-2}$ denotes the inverse of the horizontal Laplace operator. The toroidal current density can then be estimated according to equation (18):

$$
\mathbf{j}^{(\mathrm{T})} \approx-\sigma \nabla \times \hat{\mathbf{r}}\left[\nabla_{H}^{-2} \hat{\mathbf{r}} \cdot \nabla \times(\mathbf{U} \times \tilde{\mathbf{B}})\right] .
$$

Ignoring the horizontal derivatives and the cross product yields the simpler approximation

$$
\left\langle\mathbf{j}^{(T)}\right\rangle \approx \operatorname{Rm} \frac{\langle\tilde{\mathbf{B}}\rangle}{\mu d}
$$

for the rms values of the toroidal current density. In order to quantify the quality of the two estimates, we compare the ratio of the estimated to the true rms values at each radial level. The respective ratios read

$$
R_{j T}=\sigma \frac{\left\langle\nabla \times \hat{\mathbf{r}}\left[\nabla_{H}^{-2} \hat{\mathbf{r}} \cdot \nabla \times(\mathbf{U} \times \tilde{\mathbf{B}})\right]\right\rangle}{\left\langle\mathbf{j}^{(\mathrm{T})}\right\rangle},
$$


for expression (44) and

$$
R_{j T}^{\prime}=\operatorname{Rm} \frac{\langle\tilde{\mathbf{B}}\rangle}{\mu d\left\langle\mathbf{j}^{(\mathrm{T})}\right\rangle},
$$

for the simplified version (45). Figure 11a demonstrates that equation (44) does a good job in capturing the rms of the toroidal currents in the SDCR with deviations of less than 50\%. Below the SDCR, the approximation tends to overestimate the true current amplitude but nevertheless remains acceptable down to about $0.85 r_{o}$. The simplified version (45), however, severely overestimates the toroidal current density in the SDCR, indicating that the horizontal derivatives cannot be ignored in equation (44).

Perhaps more relevant is an estimate for the larger poloidal current contribution. A tempting approach is to ignore the electric potential altogether and simply assume

$$
\mathbf{j} \approx \sigma(\mathbf{U} \times \tilde{\mathbf{B}})
$$

which we refer to as the simplified Ohm's law or Ohm's law for a fast moving conductor in the following. Such an approach seems at least justified for the dominant current component, that is, for the latitudinal poloidal current that is mostly induced by fast azimuthal flows that render the electric potential contribution secondary.

For estimating the rms electrical current, we can rely on the dominant poloidal components. When ignoring the cross product, equation (48) suggests

$$
\langle\mathbf{j}\rangle \approx \operatorname{Rm} \frac{\langle\tilde{\mathbf{B}}\rangle}{\mu d} .
$$

To quantify the quality of this estimate, we calculate the ratio

$$
R_{j}=\operatorname{Rm} \frac{\langle\tilde{\mathbf{B}}\rangle}{\mu d\langle\mathbf{j}\rangle},
$$

which can be compared to the respective ratio for the integral estimate (39):

$$
R_{j}^{\prime}=\frac{\left\langle\mathscr{J}_{H}\right\rangle}{\langle\mathbf{j}\rangle}
$$

Figure 11b illustrates that the simplified Ohm's law yields a good approximation which overestimate the current density by typically less than a factor two in the SDCR. We have used magnetic Reynolds numbers based on the zonal flows in Figure 11b, which provide slightly better estimates, in particular for model G14. The integral estimate largely captures the right order of magnitude at the bottom of the SDCR where the currents are stronger, but generally seems of lower quality.

The simplified Ohm's law severely overestimates the weaker radial currents, which is likely related to a stronger electric potential contribution boosted by the radial derivative $\partial V / \partial r$. An improved estimate can be calculated from the poloidal current potential derived from the estimate for the horizontal components. Taking the horizontal divergence of equation (48) yields

$$
\nabla_{H}^{2} \frac{\partial}{\partial r} i^{(\mathrm{P})} \approx-\frac{\partial}{\partial r} j_{r} \approx \sigma \nabla_{H} \cdot(\mathbf{U} \times \mathbf{B})_{H}
$$

This suggests the integral estimate

$$
j_{r}(r) \approx \mathcal{J}_{r}(r)=\int_{r}^{r_{o}} \mathrm{~d} r^{\prime} \sigma \nabla_{H} \cdot(\mathbf{U} \times \mathbf{B})_{H} .
$$

Since the gradient in $\sigma$ dominates the integral, the expression can be simplified to

$$
j_{r} \approx-d_{\lambda} \sigma \nabla_{H} \cdot(\mathbf{U} \times \tilde{\mathbf{B}})_{H} .
$$

Ignoring the horizontal derivative and cross product yields a simplified estimate for the rms radial current: 

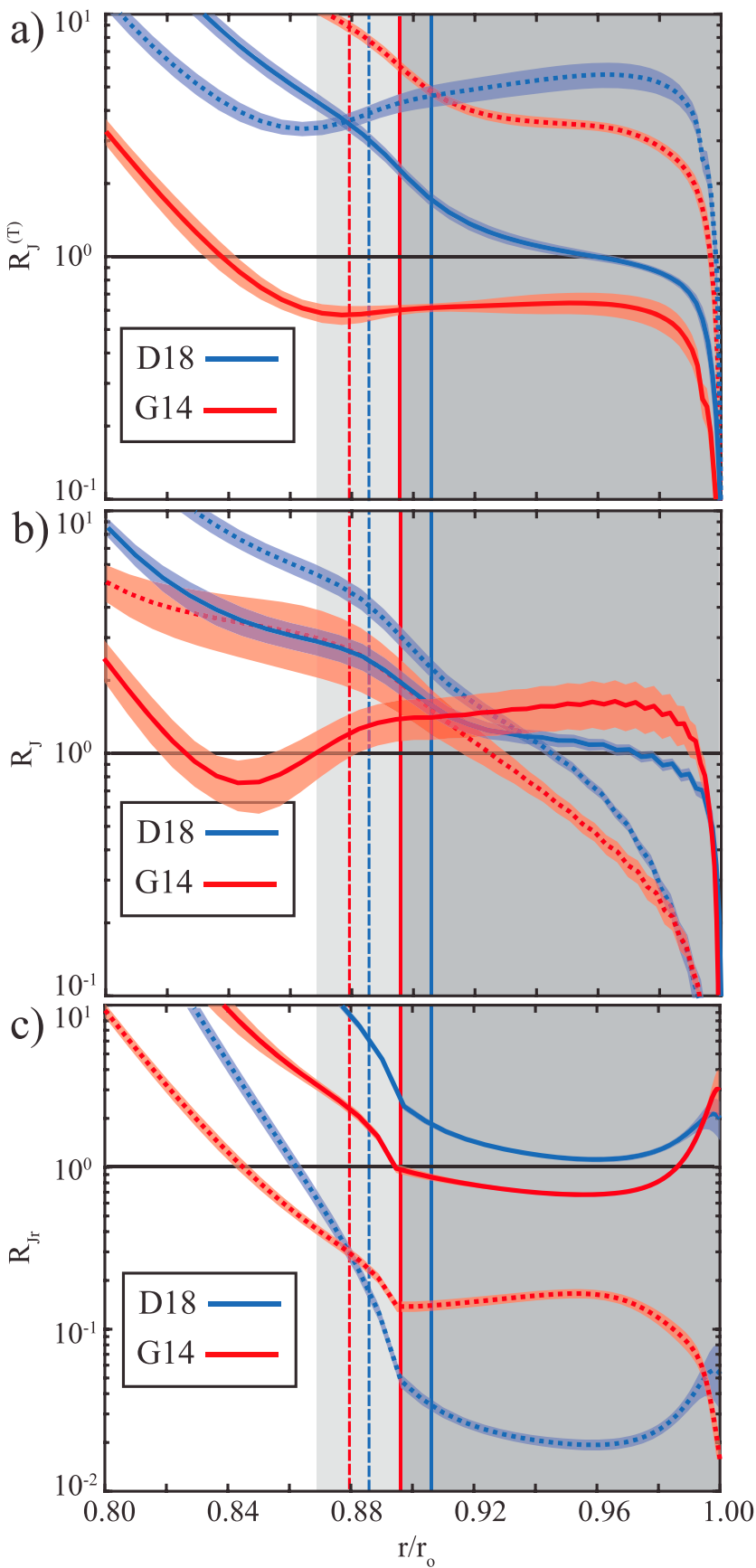

Figure 11. Ratio of estimates and true rms electric current densities. The results have been averaged over 14 snapshots for dynamo D18 and 10 snapshots for dynamo G14. Transparent background stripes with a width of two standard deviations illustrate the variability. (a) shows ratios (46) (solid lines) and (47) (dotted lines) for the toroidal currents density. (b) shows ratio (50) for the total rms current density based on Ohm's law (solid lines) and the respective ratio (51) for the integral estimate (dotted lines). Here we have used magnetic Reynolds numbers based on the zonal flows. (c) compares ratios (56) (solid lines) and (57) (dotted lines) for the radial current densities. Solid (dashed) vertical lines mark where $\mathrm{Rm}^{(1)}=1\left(\mathrm{Rm}^{(1)}=5\right)$. The medium gray background highlights the Steeply Decaying Conductivity Region where the conductivity decreases exponentially, while light gray marks the region where the conductivity is smaller than $50 \%$ of the inner-boundary reference value. 

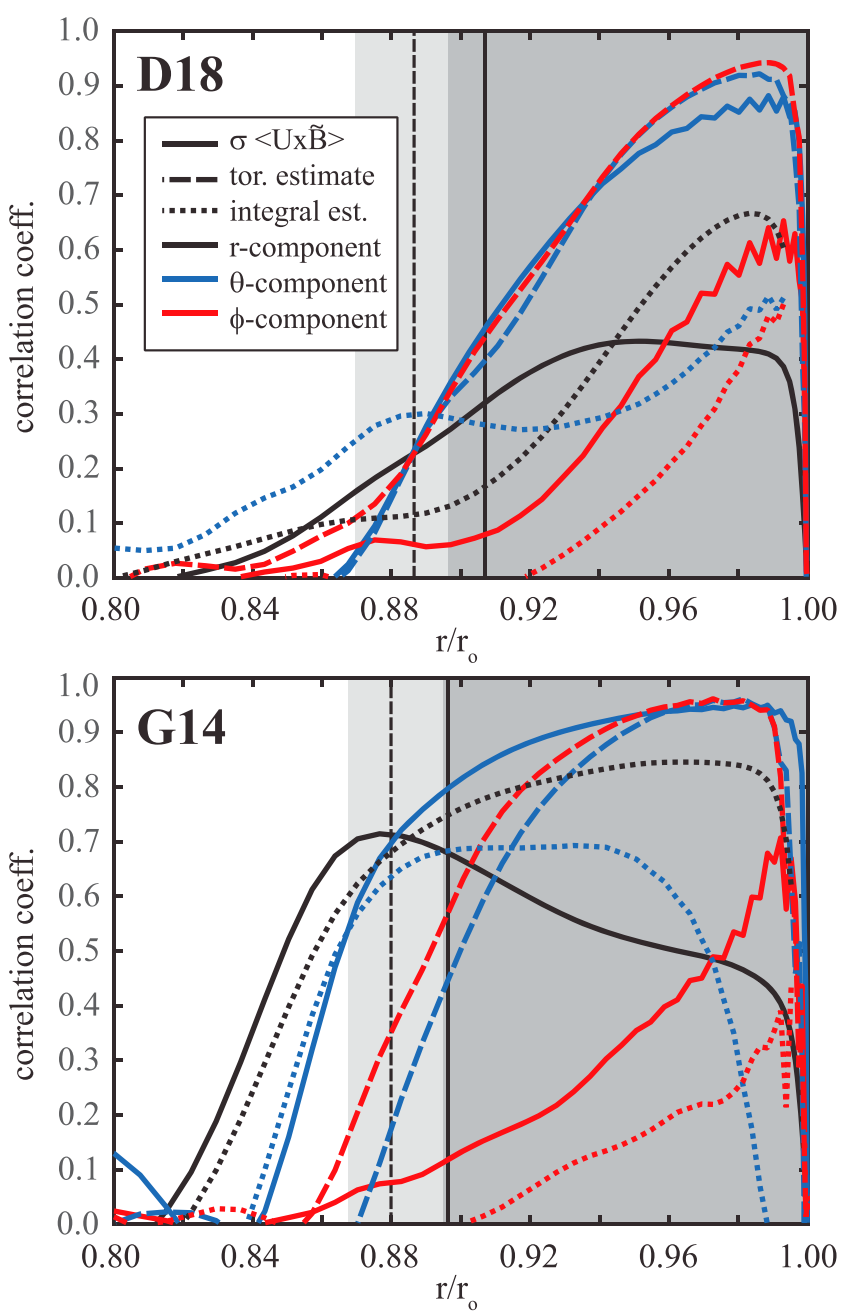

Figure 12. Pearson correlation coefficients between individual current density components and two different estimates. Solid lines refer to the estimates based on the simplified Ohm's law (48), while dotted lines refer to the integral expression (39) for the horizontal and (54) for the radial component. See caption of Figure 11 for more explanations.

$$
\left\langle j_{r}\right\rangle \approx \mathrm{Rm}^{(1)} \frac{\langle\tilde{\mathbf{B}}\rangle}{\mu d} .
$$

The quality ratio based on equation (54) reads

$$
R_{j r}=d_{\lambda} \sigma \frac{\left\langle\nabla_{H} \cdot(\mathbf{U} \times \tilde{\mathbf{B}})_{H}\right\rangle}{\left\langle j_{r}\right\rangle},
$$

while the simplified version (55) yields

$$
R_{j r}^{\prime}=\operatorname{Rm}^{(1)} \frac{\langle\tilde{\mathbf{B}}\rangle}{\mu d\langle\mathbf{j}\rangle} .
$$

Figure 11c demonstrates that the simplified version severely underestimates the radial current density by at least an order a magnitude in the SDCR, while the more complex expression (54) deviates by not more than about $30 \%$ from the true values. This confirms that the horizontal derivatives cannot be ignored.

Figure 11 suggests that the higher quality estimates remain reliable throughout the whole SDCR. The assumption of a dominant background potential field but also of the quasi-stationary dynamo action roughly holds for $\mathrm{Rm}^{(1)} \leq 1$. The radii where $\mathrm{Rm}^{(1)}=1$ and $\mathrm{Rm}^{(1)}=5$ have been marked by vertical lines in Figure 11. Some estimates provide acceptable values down to the latter radius, and the higher quality estimates of the toroidal and total current density yield acceptable values for even deeper radii. 

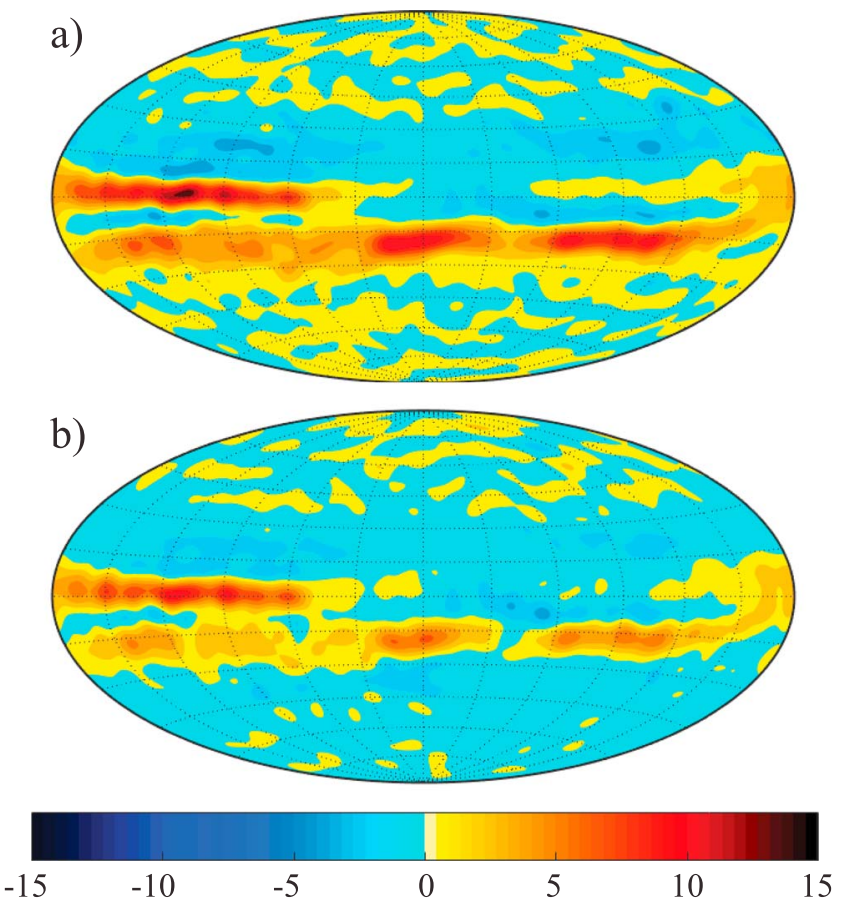

Figure 13. Comparison of (a) $\mu j_{\theta}$ with (b) estimate $\operatorname{Pm}(\mathbf{U} \times \tilde{\mathbf{B}})_{\theta}$ at $r=0.94 r_{o}$ for the snapshot of dynamo G14. Dimensionless values shown.

To assess whether the estimates of the individual current density components not only capture the rms amplitudes but also the structure, we calculate the Pearson correlation coefficients for each radius. For example, for the total latitudinal current density, the coefficient reads

$$
C\left(j_{\theta}, \sigma(\mathbf{U} \times \mathbf{B})_{\theta}\right)=\frac{\overline{\left(j_{\theta}-\bar{j}_{\theta}\right)\left([\mathbf{U} \times \mathbf{B}]_{\theta}-\overline{[\mathbf{U} \times \mathbf{B}]_{\theta}}\right)}}{\left[\overline{\left(j_{\theta}-\bar{j}_{\theta}\right)^{2}} \overline{\left([\mathbf{U} \times \mathbf{B}]_{\theta}-\overline{[\mathbf{U} \times \mathbf{B}]_{\theta}}\right)^{2}}\right]^{1 / 2}},
$$

where the overbars refer to an average over a spherical surface. The results, shown in Figure 12, demonstrate that $\sigma \mathbf{U} \times \tilde{\mathbf{B}}$ not only provides a better rms estimate but also a better local estimate than the integral estimate $\mathscr{F}_{H}$ for the horizontal current components. Particularly high correlations are reached for the toroidal and the total latitudinal current densities where the simplified Ohm's law can reasonably be applied. The inferior correlations for the azimuthal current density point toward a stronger contribution from the electric potential. For the radial component, equation (53) provides a very decent local estimate with Pearson coefficients up to 0.85 for G14. Figure 13 illustrates the close agreement for the latitudinal current density in model G14 at $r=0.94 r_{0}$. The stronger dynamo action at low to middle latitudes caused by the shear in the zonal flow is clearly apparent.

\subsection{Estimating the LIF}

Using the current provided by the simplified Ohm's law (30) for the horizontal field yields the integral estimate $\mathscr{B}_{O}$ for the horizontal LIF:

$$
\hat{\mathbf{B}}_{H} \approx \int_{r}^{r_{o}} \mathrm{~d} r \frac{\hat{\mathbf{r}} \times(\mathbf{U} \times \tilde{\mathbf{B}})}{\lambda} .
$$

This can be further simplified to

$$
\hat{\mathbf{B}}_{H} \approx \frac{d_{\lambda}}{\lambda} \hat{\mathbf{r}} \times(\mathbf{U} \times \tilde{\mathbf{B}}),
$$

since the conductivity profile dominates the radial dependence. The expression suggests a rather simple estimate for the rms value of the LIF, which is dominated by the toroidal field: 


$$
\langle\hat{\mathbf{B}}\rangle \approx \operatorname{Rm}^{(1)}\langle\tilde{\mathbf{B}}\rangle .
$$

For estimating the radial poloidal field, we rely on equation (31):

$$
\hat{B}_{r} \approx-\mu \int_{r}^{r_{o}} \mathrm{~d} r^{\prime} \int_{r^{\prime}}^{r_{o}} \mathrm{~d} r^{\prime \prime} \sigma \hat{\mathbf{r}} \cdot\left(\nabla^{\prime \prime} \times[\mathbf{U} \times \tilde{\mathbf{B}}]\right) \approx-\frac{d_{\lambda}^{2}}{\lambda} \hat{\mathbf{r}} \cdot \nabla \times(\mathbf{U} \times \tilde{\mathbf{B}}) .
$$

This is equivalent to assuming a balance between induction and diffusion in the induction equation (35) and approximating the latter term with $\lambda / d_{\lambda}^{2} \hat{B}_{r}$.

Ignoring the curl and cross product suggests that the rms value of the radial LIF can be estimated via

$$
\left\langle\hat{\mathrm{B}}_{r}\right\rangle \approx \mathrm{Rm}^{(2)}\langle\tilde{\mathbf{B}}\rangle \text {. }
$$

Taking the radial derivative of equation (62) yields a differential equation for the horizontal poloidal LIF:

$$
\frac{\partial}{\partial r} \hat{B}_{r} \approx-\nabla_{H}^{2} \frac{\partial}{\partial r} \hat{b}^{(\mathrm{P})}=-\nabla_{H} \hat{\mathbf{B}}_{H}^{(\mathrm{P})} \approx \frac{d_{\lambda}}{\lambda} \hat{\mathbf{r}} \cdot \nabla \times(\mathbf{U} \times \tilde{\mathbf{B}}) .
$$

We solve this equation by taking the horizontal derivatives in spectral space and formally write the result as

$$
\hat{\mathbf{B}}_{H}^{(\mathrm{P})} \approx-\frac{d_{\lambda}}{\lambda} \nabla_{H}^{-1} \hat{\mathbf{r}} \cdot \nabla \times(\mathbf{U} \times \tilde{\mathbf{B}}),
$$

where $\nabla_{H}^{-1}$ denotes the inverse horizontal nabla operator. Ignoring once more cross product and horizontal derivatives suggests the simplified estimate

$$
\left\langle\hat{\mathrm{B}}_{H}^{(\mathrm{P})}\right\rangle \approx \mathrm{Rm}^{(1)}\langle\tilde{\mathbf{B}}\rangle
$$

for the rms amplitude of the horizontal poloidal LIF. This would imply that poloidal and toroidal LIF are comparable in amplitude, but we already know from section 3 that the toroidal field dominates.

We quantify the quality of the LIF estimates (61)-(63), (65), and (66) with the ratios

$$
\begin{gathered}
R_{B}=\operatorname{Rm}^{(1)} \frac{\langle\tilde{\mathrm{B}}\rangle}{\left\langle\hat{\mathbf{B}}^{(\mathrm{P})}\right\rangle}, \\
R_{B r}=\frac{d_{\lambda}^{2}}{\lambda} \frac{\langle\hat{\mathbf{r}} \cdot \nabla \times(\mathbf{U} \times \tilde{\mathbf{B}})\rangle}{\left\langle\hat{\mathrm{B}}_{r}\right\rangle}, \\
R_{B r}^{\prime}=\operatorname{Rm}^{(2)} \frac{\langle\tilde{\mathbf{B}}\rangle}{\left\langle\hat{B}_{r}\right\rangle}, \\
R_{B H}^{(\mathrm{P})}=\frac{d_{\lambda}}{\lambda} \frac{\left\langle\nabla_{H}^{-1} \hat{\mathbf{r}} \cdot \nabla \times(\mathbf{U} \times \tilde{\mathbf{B}})\right\rangle}{\left\langle\hat{\mathrm{B}}_{r}\right\rangle},
\end{gathered}
$$

and

$$
R_{B H}^{(\mathrm{P})}=\operatorname{Rm}^{(1)} \frac{\langle\tilde{\mathbf{B}}\rangle}{\left\langle\hat{\mathbf{B}}_{h}^{(\mathrm{P})}\right\rangle},
$$

respectively.

Figure 14 shows that very reasonable values between $R_{B}=1.3$ and $R_{B}=2$ for the total LIF, between $R_{B r}=0.8$ and $R_{B r}=2$ for the radial components, and between $B_{B H}^{(P)}=0.4$ and $R_{B H}^{(P)}=1.3$ for the horizontal poloidal components can be achieved in the SDCR, ignoring once more the very outer few percent in radius. The region where the estimates offer acceptable results extends to a depth between $\mathrm{Rm}^{(1)}=1$ and $\mathrm{Rm}^{(1)}=5$ (see vertical lines in Figure 14). The simplified estimates (69) and (71) underestimates the radial component and severely overestimate the horizontal poloidal components. This once more highlights that ignoring the horizontal derivatives leads to inferior estimates. 


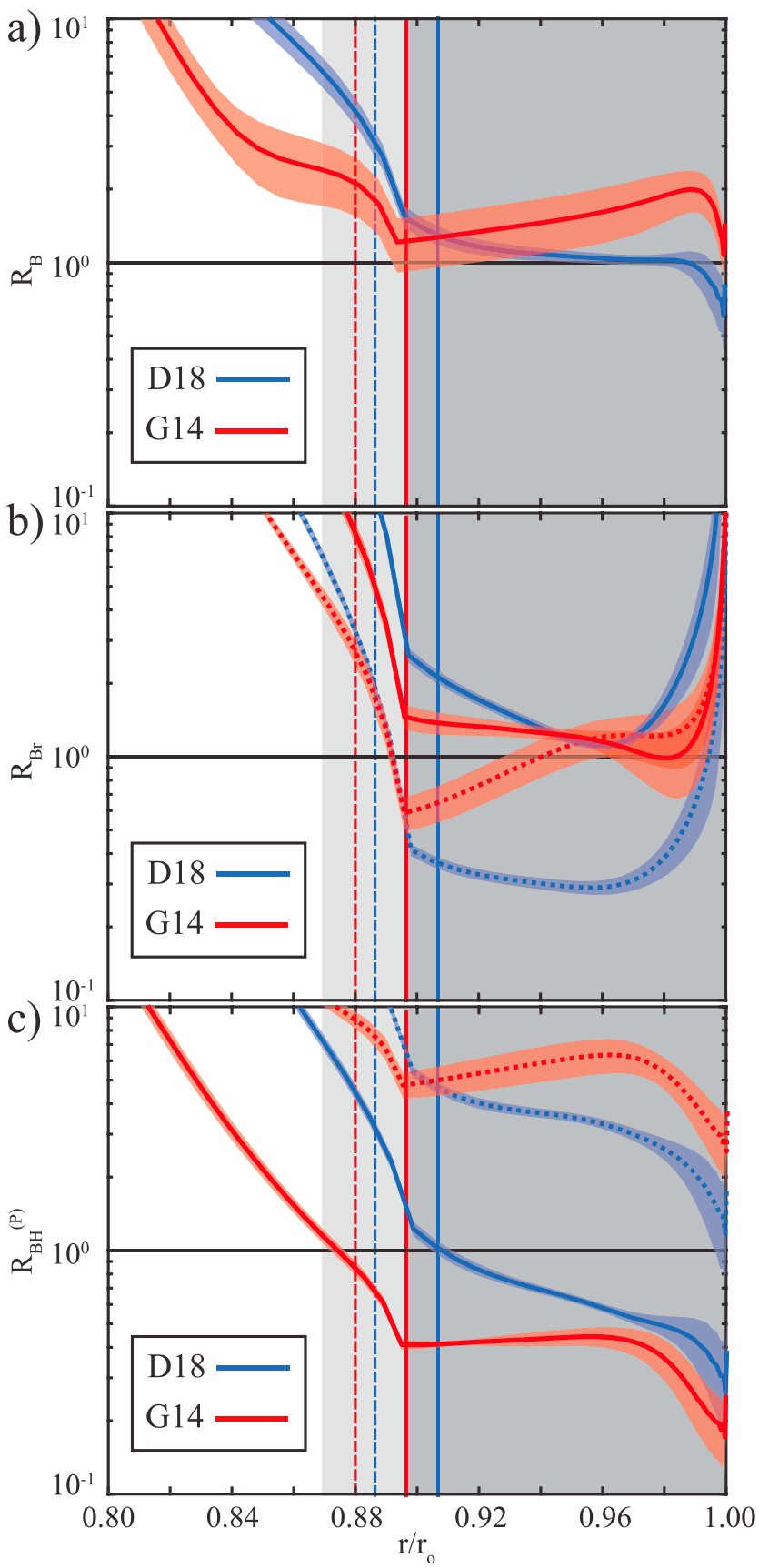

Figure 14. Ratio of rms values for the different Locally Induced Field estimates and respective true values. (a) shows the ratio for the total LIF estimate (67). Zonal flow magnetic Reynolds numbers have been used. (b) compares the ratios for the more complex radial field estimate (62) (solid lines) with the simplified version (63) (dotted lines). (c) compares the ratio for the more complex horizontal poloidal field estimate (65) (solid lines) with the respective simplified version (66) (dotted lines). See caption of Figure 11 for more explanations.

Figure 15 shows the Pearson correlation coefficients for the individual LIF components based on estimates (61), (62), and (65). In the region where $\mathrm{Rm}^{(1)} \geq 1$, that is, to the right of the solid vertical lines, the coefficients range between 0.4 and 0.93 for the radial LIF and between 0.45 and 0.95 for the total azimuthal component. These high values reflect the fact that the simplified Ohm's law holds to a larger degree for the underlying current components. The poloidal LIF depends on the toroidal current density, and the total azimuthal LIF is mostly determined by the latitudinal current component. Based on these arguments, we would also expect high correlation coefficients for the horizontal poloidal LIF. However, this is only true for 

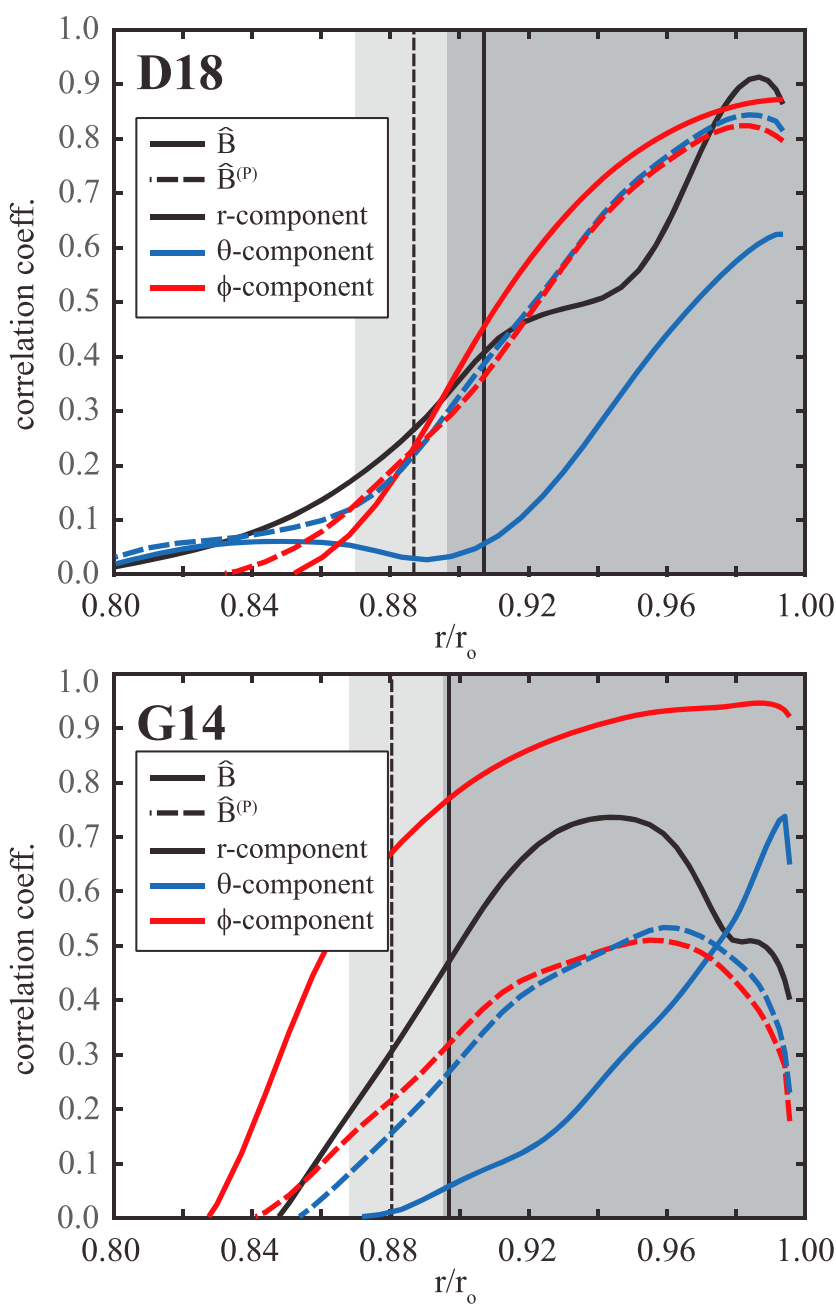

Figure 15. Pearson correlation coefficients between individual magnetic field components and estimates (60) for the horizontal, (62) for the radial, and (65) for the horizontal poloidal field. See caption of Figure 11 for more explanation.

model D18, while the values for model G14 hardly exceeds 0.5 . A possible reasons are the complex horizontal derivatives involved in the respective estimate (65). The lower quality of the total latitudinal LIF estimate, on the other hand, is a consequence of the inferior azimuthal poloidal current estimate (see above).

Figure 16 illustrates the good agreement between $\hat{\mathrm{B}}_{r}$ and estimate (62) at $0.94 r_{o}$ for the G14 snapshot. The stronger toroidal field induced by the shear in the zonal flow also leads to a stronger radial LIF in a band around the equator. Its small scale reflects the complex nonlinear interaction between the background field and the local flow. The more banded structures described by Gastine, Wicht, et al. (2014) are created below the SDCR, where the zonal flows are weaker but the related magnetic Reynolds number nevertheless higher.

\subsection{Estimating the Lorentz Force}

Having derived estimates for electric currents and magnetic fields, we can combine both to assess the Lorentz force $\mathbf{L}$ in the SDCR. Of particular interest is its zonal (axisymmetric azimuthal) component $\bar{L}_{\phi}$, which could potentially impact the zonal winds. Figure 17 shows the profiles of the rms $\bar{L}_{\phi}$ for dynamos D18 and G14. From the two contributions,

$$
\bar{L}_{\phi}=\overline{j_{r} B_{\theta}}-\overline{j_{\theta} B_{r}}
$$

the second clearly dominates because of the higher latitudinal current density.

Combining the estimates for $j_{\theta}$ and $B_{r}$ then suggest 
a)

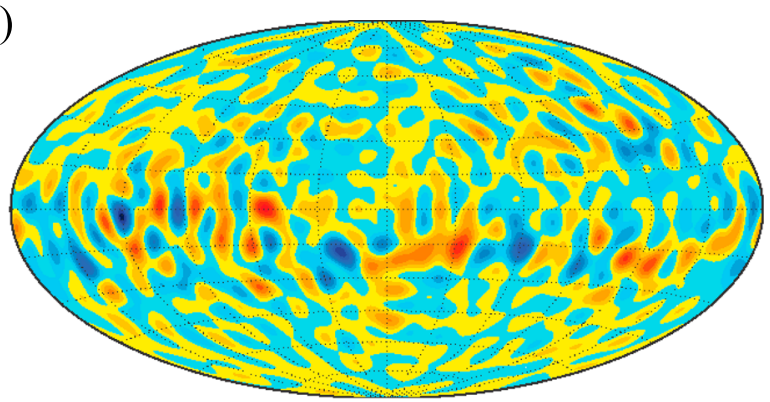

b)
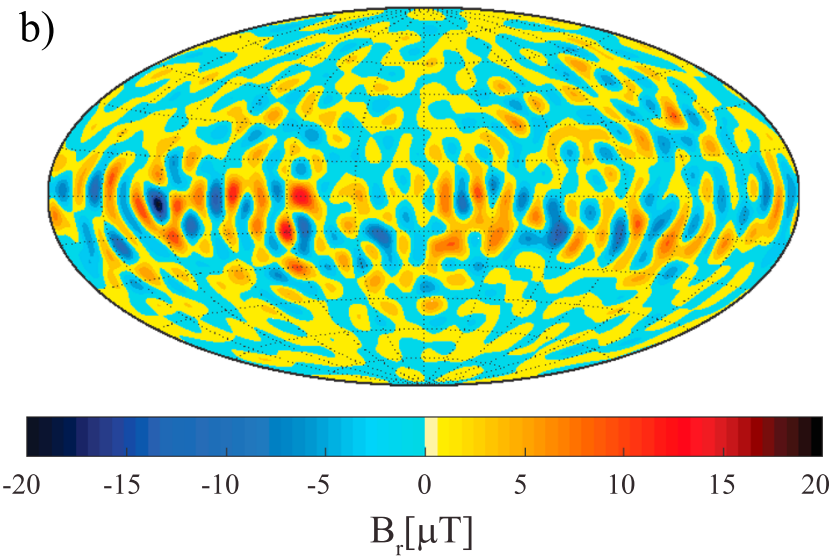

Figure 16. Comparison of (a) the radial Locally Induced Field $\hat{\mathrm{B}}_{r}$ with (b) the estimate (62) at $r=0.94 r_{o}$ for the dynamo G14 snapshot. The fields have been rescaled to dimensional values and can directly be compared with the surface field shown in Figure 2.

$$
\bar{L}_{\phi} \approx-\sigma \overline{(\mathbf{U} \times \mathbf{B})_{\theta} \mathrm{B}_{r}}=-\sigma \overline{\mathrm{U}_{\phi} \mathrm{B}_{r}^{2}}+\sigma \overline{\mathrm{U}_{r} \mathrm{~B}_{\phi} \mathrm{B}_{r}} .
$$

Figure 17 illustrates that the first contribution dominates, in particular for model G14 where zonal flows are stronger. We can thus estimate the rms zonal Lorentz force based on a conductivity model, a zonal flow model, and the background field:

$$
\left\langle\bar{L}_{\phi}\right\rangle \approx \sigma\left\langle\overline{\mathrm{U}}_{\phi}\right\rangle\left\langle\overline{\tilde{\mathrm{B}}_{r}^{2}}\right\rangle .
$$

Figure 18 shows the respective ratio,

$$
R_{L F}=\frac{\sigma\left\langle\overline{\mathrm{U}}_{\phi}\right\rangle\left\langle\overline{\mathrm{B}}_{r}^{2}\right\rangle}{\left\langle\bar{L}_{\phi}\right\rangle},
$$

and demonstrates that the expression tends to underestimate the zonal Lorentz force in the SDCR by up to $40 \%$. The region where this estimate can reasonable be applied is larger for G14 where zonal flows are stronger.

\section{Discussion and Conclusion}

The analysis of our numerical simulations confirms that dynamo action in the SDCR is dominated by Ohmic dissipation. The magnetic field dynamics becomes quasi-stationary with a good balance between induction and diffusion. Ohm's law, on the other hand, assumes the simplified form for a fast moving conductor for the most relevant current contributions where the electric field contribution can be neglected. Electric currents and the toroidal field simply decay with the electrical conductivity, while the poloidal field approaches a potential field.

This particular situation allows to formulate rather simple estimates based on the knowledge of the surface field, the conductivity profile, and the flow. The electric current density can be estimated via the simplified 

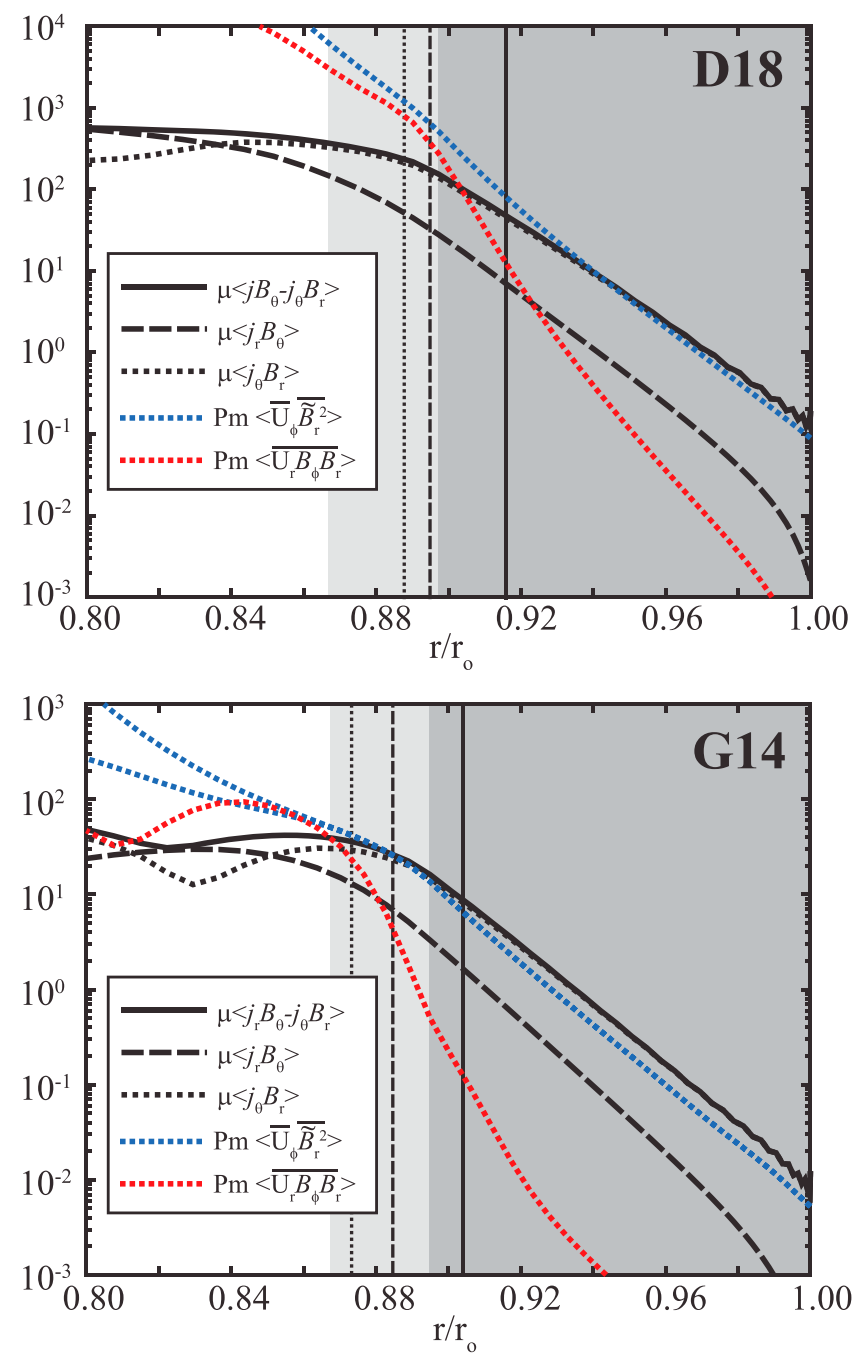

Figure 17. Zonal rms Lorentz force contributions and estimates. See text and caption of Figure 11 for more explanations.

Ohm's law with a suggested rms value of $\langle\mathbf{J}\rangle \approx \sigma\langle\mathbf{U}\rangle\langle\tilde{\mathbf{B}}\rangle$, where $\tilde{\mathbf{B}}$ is the downward continued surface field under the potential field assumption. When applied to the numerical simulations, the accuracy is higher than the more classical estimate used by Liu et al. (2008) for their assessment of Ohmic heating due to Jupiter's zonal winds.

Also of interest is the radial LIF that could, for example, potentially be detected by the Juno spacecraft. Our analysis shows that $\left\langle\mathrm{B}_{r}^{\prime}\right\rangle \approx \mathrm{Rm}^{(2)}\langle\tilde{\mathbf{B}}\rangle$ provides a rough but somewhat too low estimate. Here $\mathrm{Rm}^{(2)}$ is a modified magnetic Reynolds number that depends on the square of the diffusivity scale height $d_{\lambda}$. The toroidal LIF, on the other hand, can be predicted via $\langle\mathbf{B}\rangle \approx \mathrm{Rm}^{(1)}\langle\tilde{\mathbf{B}}\rangle$ and is thus by up to a factor $\mathrm{Rm}^{(1)} / \mathrm{Rm}^{(2)}=d / d_{\lambda}$ larger than the radial field, where $d$ is the depth of the dynamo region. When using the conductivity model by French et al. (2012), this predicts that the toroidal LIF is about $10^{2}$ to $10^{3}$ time larger than the radial LIF at $0.96 r_{J}$ in Jupiter. At $0.9 r_{J}$, this ratio has decreased by about one order of magnitude.

While the toroidal field estimate agrees with the assessment by Cao and Stevenson (2017), the poloidal field estimates differ. Cao and Stevenson (2017) consider the poloidal field produced by nonaxisymmetric (helical) flows acting on the local toroidal field, but the advective modification of $\tilde{\mathbf{B}}$ turns out to be significantly larger in the SDCR of our simulations.

Duarte et al. (2018) define the radius $r_{D}$ for the top of the dynamo region based on a critical magnetic Reynolds number. A more reasonable local definition is the depth where the local magnetic effects reach a 


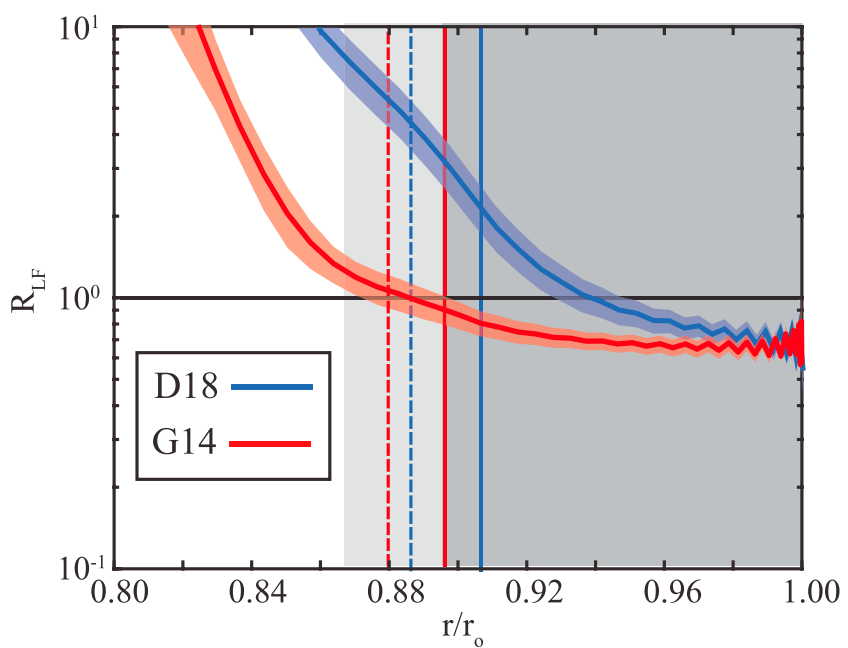

Figure 18. Ratio of estimate (74) for the zonal Lorentz force and the true value. See caption of Figure 11 for more explanations. certain threshold. Since the electric current, toroidal field, and poloidal field all scale with different magnetic Reynolds numbers, the answer will actually depend on the considered quantity.

Choosing the poloidal magnetic field has the advantages that it can actually be observed. For the most recent field models based on Juno data, Connerney et al. (2018) discuss a value of $r_{D}=0.85 r_{J}$. The authors argue that the spectrum of $\tilde{\mathbf{B}}$ is close to a white spectrum at this depth, a criterion that successfully predicts Earth's core-mantle boundary when excluding the axial dipole contribution. However, $0.85 r_{J}$ lies below the transition to the metallic Hydrogen region (French et al., 2012) and below the anticipated depth of the zonal winds (Kaspi et al., 2018). The magnetic Reynolds numbers reached in the simulations are orders of magnitude lower than in Jupiter (Duarte et al., 2018). Moreover, they decrease significantly less steeply with radius because of the more moderate conductivity profile (see Figure 1). When adopting a mean convective velocity of $3 \mathrm{~cm} / \mathrm{s}$ (Gastine, Wicht, et al., 2014) and the French conductivity profile (French et al., 2012), Jovian magnetic Reynolds number profiles reach $\mathrm{Rm}^{(1)}=$ $2.7 \times 10^{4}$ and $\mathrm{Rm}=7.6 \times 10^{5}$ at $0.85 r_{J}$, while the values in the numerical simulations are only of order $10^{2}$ and 10 , respectively.

The huge Jovian values suggest that the dynamo action should already be more than well developed at $0.85 r_{J}$. Our convective-flow based rendition of Jupiter's $\mathrm{Rm}^{(1)}$ profile actually already exceeds unity at about $0.93 r_{J}$, which is therefore roughly the depth where the estimates discussed here start to lose their basis. Since $\mathrm{Rm}^{(2)}$ is about $10^{-4}$ at $0.93 r_{J}$ and the potential field amplitude is about $1 \mathrm{mT}$, the radial LIF would be roughly $10^{-3}$ to $10^{-4} \mathrm{mT}$.

However, these considerations ignore the zonal winds, which reach amplitudes of around $150 \mathrm{~m} / \mathrm{s}$ at Jupiter's cloud level. Recent considerations based on Juno's gravity measurements constrain the depth profile of the zonal winds and suggest a considerably lower wind speed at about $0.96 r_{J}$. The estimates derived here, in combination with the new depth profiles for the zonal winds, should allow predicting the zonal flow induced magnetic fields, electric currents and the related Ohmic heating. The analysis presented here suggest that decent local estimates are also possible. The predicted maps of Ohmic heating and radial field modifications could be compared with spacecraft observation to detect potential zonal field induction effects.

The questions addressed here are also of interest for other planets where fast fluid flows exist in a region of decaying electrical conductivity. Saturn, Uranus, and Neptune come to mind. The Ohmic heating in the outer atmospheres of hot Jupiters, where the ionization of alkali metals creates a SDCR, is a possible mechanism to explain the particularly low density (inflation) of some of these exoplanets. Assuming a quasi-stationary induction equation, Batygin and Stevenson (2010) conclude that the locally induced currents would indeed provide sufficient heating power. Our result suggest that their approach and thus also likely their conclusions are viable.

\section{Acknowledgments}

This work was supported by the German Research Foundation (DFG) in the framework of the special priority program PlanetMag (SPP 1488). The MHD-code MagIC used for the simulations is freely available on GitHub (https://github.com/ magic-sph/magic). The simulation data for the two cases expored here and the Matlab package used for the analysis can be downloaded online (https://dx.doi.org/10.17617/3.1q)

\section{References}

Batygin, K., \& Stevenson, D. J. (2010). Inflating hot Jupiters with Ohmic dissipation. Astrophysical Journal, 714, L238-L243. https://doi.org/10.1088/2041-8205/714/2/L238

Braginsky, S., \& Roberts, P. (1995). Equations governing convection in Earth's core and the geodynamo. Geophysical and Astrophysical Fluid Dynamics, 79, 1-97.

Cao, H., \& Stevenson, D. J. (2017). Gravity and zonal flows of giant planets: From the Euler equation to the thermal wind equation. Journal of Geophysical Research: Planets, 122, 686-700. https://doi.org/10.1002/2017JE005272

Christensen, U., \& Aubert, J. (2006). Scaling properties of convection-driven dynamos in rotating spherical shells and applications to planetary magnetic fields. Geophysical Journal International, 116, 97-114.

Christensen, U., \& Wicht, J. (2007). Numerical dynamo simulations (2nd ed.). In O. P. (Ed.), Core dynamics, treatise on geophysics (Vol. 8, pp. 245-282). Oxford: Elsevier.

Connerney, J. E. P., Acuña, M. H., Ness, N. F., \& Satoh, T. (1998). New models of Jupiter's magnetic field constrained by the Io flux tube footprint. Journal of Geophysical Research, 103, 11,929-11,940. https://doi.org/10.1029/97JA03726

Connerney, J. E. P., Kotsiaros, S., Oliversen, R. J., Espley, J. R., Joergensen, J. L., Joergensen, P. S., et al. (2018). A new model of Jupiter's magnetic field from Juno's first nine orbits. Geophysical Research Letters, 45, 2590-2596. https://doi.org/10.1002/2018GL077312 
Duarte, L. D. V., Gastine, T., \& Wicht, J. (2013). Anelastic dynamo models with variable electrical conductivity: An application to gas giants. Physics of the Earth and Planetary Interiors, 222, 22-34. https://doi.org/10.1016/j.pepi.2013.06.010

Duarte, L. D. V., Wicht, J., \& Gastine, T. (2018). Physical conditions for Jupiter-like dynamo models. Icarus, 299, 206-221. https://doi.org/10.1016/j.icarus.2017.07.016

French, M., Becker, A., Lorenzen, W., Nettelmann, N., Bethkenhagen, M., Wicht, J., \& Redmer, R. (2012). Ab initio simulations for material properties along the Jupiter adiabat. Astrophysical Journal Supplement, 202, 5. https://doi.org/10.1088/0067-0049/202/1/5

Gastine, T., Duarte, L., \& Wicht, J. (2012). Dipolar versus multipolar dynamos: The influence of the background density stratification. Astronomy \& Astrophysics, 546, A19.

Gastine, T., Heimpel, M., \& Wicht, J. (2014). Zonal flow scaling in rapidly-rotating compressible convection. Physics of the Earth and Planetary Interiors, 232, 36-50. https://doi.org/10.1016/j.pepi.2014.03.011

Gastine, T., \& Wicht, J. (2012). Effects of compressibility on driving zonal flow in gas giants. Icarus, 219, 428-442. https://doi.org/10.1016/j.icarus.2012.03.018

Gastine, T., Wicht, J., Duarte, L. D. V., Heimpel, M., \& Becker, A. (2014). Explaining Jupiter's magnetic field and equatorial jet dynamics. Geophysical Research Letters, 41, 5410-5419. https://doi.org/10.1002/2014GL060814

Guillot, T., Miguel, Y., Militzer, B., Hubbard, W. B., Kaspi, Y., Galanti, E., et al. (2018). A suppression of differential rotation in Jupiter's deep interior. Nature, 555, 227-230. https://doi.org/10.1038/nature25775

Iess, L., Folkner, W. M., Durante, D., Parisi, M., Kaspi, Y., Galanti, E., et al. (2018). Measurement of Jupiter's asymmetric gravity field. Nature, 555, 220-222. https://doi.org/10.1038/nature25776

Jones, C. A. (2014). A dynamo model of Jupiter's magnetic field. Icarus, 241, 148-159. https://doi.org/10.1016/j.icarus.2014.06.020

Jones, C. A., Boronski, P., Brun, A. S., Glatzmaier, G. A., Gastine, T., Miesch, M. S., \& Wicht, J. (2011). Anelastic convection-driven dynamo benchmarks. Icarus, 216, 120-135. https://doi.org/10.1016/j.icarus.2011.08.014

Kaspi, Y., Galanti, E., Hubbard, W. B., Stevenson, D. J., Bolton, S. J., Iess, L., et al. (2018). Jupiter's atmospheric jet streams extend thousands of kilometres deep. Nature, 555, 223-226. https://doi.org/10.1038/nature25793

Kong, D., Zhang, K., Schubert, G., \& Anderson, J. D. (2018). Origin of Jupiters cloud-level zonal winds remains a puzzle even after Juno. Proceedings of the National Academy of Sciences of the United States of America, 115, 8499-8504.

Lantz, S. R., \& Fan, Y. (1999). Anelastic magnetohydrodynamic equations for modeling solar and stellar convection zones. Astrophysical Journal Supplement, 121, 247-264. https://doi.org/10.1086/313187

Liu, J., Goldreich, P. M., \& Stevenson, D. J. (2008). Constraints on deep-seated zonal winds inside Jupiter and Saturn. Icarus, 196, 653-664. https://doi.org/10.1016/j.icarus.2007.11.036

Moore, K. M., Yadav, R. K., Kulowski, L., Cao, H., Bloxham, J., Connerney, J. E. P., et al. (2018). A complex dynamo inferred from the hemispheric dichotomy of Jupiter's magnetic field. Nature, 561, 76-78. https://doi.org/10.1038/s41586-018-0468-5

Nettelmann, N., Becker, A., Holst, B., \& Redmer, R. (2012). Jupiter models with improved ab initio hydrogen equation of state (H-REOS.2). Astrophysical Journal, 750, 52. https://doi.org/10.1088/0004-637X/750/1/52

Ridley, V. A., \& Holme, R. (2016). Modeling the Jovian magnetic field and its secular variation using all available magnetic field observations. Journal of Geophysical Research: Planets, 121, 309-337. https://doi.org/10.1002/2015JE004951

Wicht, J., French, M., Stellmach, S., Nettelmann, N., Gastine, T., Duarte, L., \& Redmer, R. (2018). Modeling the interior dynamics of gas planets. In H. Lühr, J. Wicht, S. A. Gilder, \& M. Holschneider (Eds.), Magnetic fields in the solar system (pp. 7-81). Switzerland: Springer Cham 